\title{
Intermolecular Recognition of the Antimalarial Drug Chloroquine: A Quantum Theory of Atoms in Molecules-Density Functional Theory Investigation of the Hydrated Dihydrogen Phosphate Salt from the 103 K X-ray Structure
}

\author{
Giovanni Macetti, ${ }^{\dagger,}$ Laura Loconte, ${ }^{\dagger}$ Silvia Rizzato, ${ }^{\dagger}$ Carlo Gatti, ${ }^{\ddagger}$, and Leonardo Lo Presti $^{*}, \dagger, \ddagger$ \\ ${ }^{\dagger}$ Chemistry Department, Università degli Studi di Milano, Via Golgi 19, 20133 Milano, Italy \\ ${ }^{\ddagger}$ Center for Materials Crystallography, Århus Universitët, Langelandsgade 140, 8000, Århus, Denmark \\ ${ }^{\S}$ CNR-ISTM, Via Golgi 19, 20133 Milano, Italy
}

Supporting Information

ABSTRACT: The relevant noncovalent interaction patterns responsible for intermolecular recognition of the antiplasmodial chloroquine (CQ) in its bioactive diprotonated form, $\mathrm{CQH}_{2}{ }^{2+}$, are investigated. Chloroquine dihydrogen phosphate hydrated salt $\left(P 2_{1} / c\right)$ was crystallized by gel diffusion. A highresolution single-crystal $\mathrm{X}$-ray diffraction experiment was performed at $103(2) \mathrm{K}$, and a density functional theory model for the in-crystal electron density was derived, allowing the estimation of the interaction energies in relevant molecular

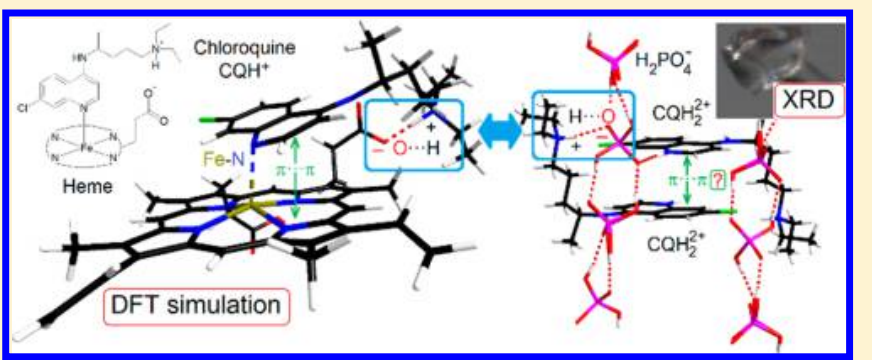
pairs. $\mathrm{H}_{2} \mathrm{PO}_{4}^{-}$ions form infinite chains parallel to the monoclinic axis, setting up strong $\mathrm{NH} \cdots \mathrm{O}$ charge-assisted hydrogen bonds (CAHBs) with $\mathrm{CQH}_{2}{ }^{2+}$. Couples of facing protonated quinoline rings are packed in a $\pi \cdots \pi$ stacked arrangement, whose contribution to the interaction energy is very low in the crystal and completely overwhelmed by Coulomb repulsion between positive aromatic rings. This questions the ability of CQ in setting up similar stacking interactions with the positively charged Fe-protoporphyrin moiety of the heme substrate in solution. When the heme/CQ adduct incorporates a $\mathrm{Fe}-\mathrm{N}$ coordinative bond, stronger $\pi \cdots \pi$ interactions are instead established due to the lacking of net electrostatic repulsions. Yet, CAHBs among the protonated tertiary amine of CQ and the propionate group of heme still provide the leading stabilizing effect. Implications on possible modifications/improvements of the CQ pharmacophore are discussed.

\section{INTRODUCTION}

Chloroquine (CQ Scheme 1) belongs to the class of 4aminoquinoline (4-AQ) antiplasmodials, whose charged forms

Scheme 1

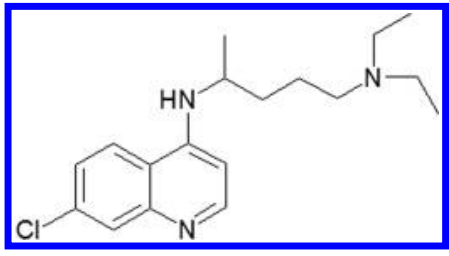

are concentrated in vivo within the acidic digestive vacuole (DV) of the Plasmodium protozoa, the malaria parasites. ${ }^{1}$ In the $\mathrm{DV}$, these drugs interfere with the detoxification process of the free heme released by digestion of hemoglobin, ${ }^{2}$ probably by acting as biocrystallization inhibitors of hemozoin ( $\beta$-hematin), the heme crystals. ${ }^{1,3-5}$ In the presence of 4-AQ compounds, free heme is released into the cytosol, where it increases the cellular oxidative stress. ${ }^{6,7}$ It is accepted that the formation of some kind of heme-drug complex lies at the core of the antimalarial activity of 4-AQ drugs, including CQ. There is also a large consensus, substantiated by $\mathrm{UV}^{8}$ extended $\mathrm{X}$-ray absorption fine structure (EXAFS), ${ }^{9} \mathrm{NMR}^{3} \mathrm{MM}^{3,10}$ and density functional theory (DFT) ${ }^{11}$ findings, on the relevant role of $\pi \cdots \pi$ stacking interactions between the quinoline core of the drug and the heme pyrrole subunits to stabilize such a complex. However, no conclusive evidence has been provided to account for the most probable structure of the adduct. In a recent work, ${ }^{12}$ in agreement with former NMR solid-state findings, ${ }^{4}$ we found that a direct $\mathrm{Fe}-\mathrm{N}$ (quinoline) coordinative bond might be also established in solution. These two heme/CQ recognition modes $(\pi \cdots \pi$ stacking or $\mathrm{Fe}-\mathrm{N})$ are usually believed to be mutually exclusive. ${ }^{13}$

The main purpose of this work is to shed light on this issue. Because of the well-known difficulties in gaining insights into the molecular recognition process in solution, we undertake a high-resolution X-ray diffraction experiment on the dihydrogen

Received: July 19, 2016

Revised: September 7, 2016

Published: September 21, 2016 
phosphate dihydrate salt of diprotonated chloroquine, $\mathrm{CQH}_{2}{ }^{2+}$ (Sections 2 and 3.1). A comprehensive analysis of the most important packing features is carried out on the basis of the interaction energies of molecular pairs and of the properties of their corresponding electron density (ED) distributions. The most striking structure-determining noncovalent interactions (NCI) (Section 3.2) are singled out and correlated, at least qualitatively, with the predicted aspects of the heme/CQ interaction mode (Section 3.3). To this end, dispersioncorrected DFT calculations were employed to compute a reasonable structure for the heme/CQ adduct, possibly to be able to reconciliate the available, yet sometimes apparently contradictory, experimental evidence. In Section 4, the main findings are summarized and discussed in view of developing novel effective cheap CQ-based antiplasmodials, able to counteract the evolved resistance of Plasmodium falciparum, the most virulent malaria parasite. ${ }^{14,15}$

\section{MATERIALS AND METHODS}

2.1. Crystal Growth and X-ray Data Collection. White powder of reagent-grade anhydrous $N^{4}$-(7-chloro-4-quinolinyl)- $N^{\prime}, N^{\prime}$-diethyl1,4-pentanediamine (chloroquine) diphosphate salt (Sigma-Aldrich) was used without further purification. Single crystals of the dihydrate salt were grown by sol-gel diffusion $(0.3 \mathrm{M}$ solution in distilled water against THF as the antisolvent) within a glass tube (ø $12 \mathrm{~mm})$, using agarose $(1 \% \mathrm{~m} / \mathrm{v})$ as a gelling agent. ${ }^{16}$ Single-crystal X-ray diffraction (SC-XRD) data were collected both at room temperature (RT) and at $T=103(2) \mathrm{K}$ on a three-circle Bruker Apex II CCD diffractometer equipped with an Oxford Cryosystems $\mathrm{N}_{2}$ gas blower, at a nominal source power of $50 \mathrm{kV} \times 30 \mathrm{~mA}$. $100 \%$ complete data sets, up to a maximum resolution of $\sin \theta / \lambda=0.6 \AA^{-1}(\mathrm{RT})$ and $1.0 \AA^{-1}(103 \mathrm{~K})$, were obtained. Integration and preliminary data reduction were performed using SAINT, ${ }^{17}$ while empirical absorption correction and scaling were performed by SADABS ${ }^{18}$ and XPREP. ${ }^{19}$ The crystal structure was solved by direct methods (SHELXS-2014) ${ }^{20}$ and refined by least-squares against $\mathrm{F}^{2}$ (SHELXL-2014) ${ }^{20}$ Relevant refinement details and agreement statistics can be found in Table 1; CCDC $1471834(103 \mathrm{~K})$ and 1494003 (RT) contain the supplementary crystallographic data for this paper. These data are provided free of charge by the Cambridge Crystallographic Data Centre.

2.2. Solid-State DFT Simulations. Periodic DFT (P-DFT) optimization of the experimental structure was performed within a linear combination of Gaussian-type function (LCGTF) approach, as implemented in the CRYSTAL14 code, ${ }^{21}$ at the 6-31G(d) B3LYP theory level. Cell parameters and crystal symmetries were kept fixed during the whole optimization process to those provided by the SCXRD experiment at $103 \mathrm{~K}$ (Table 1). The interested reader can find full technical details on the energy and geometry minimization procedures in the Supporting Information (Section S1). Topological analysis of the periodic ED, $\rho(\mathbf{r})$, was carried out through the TOPOND $^{12,22}$ module of CRYSTAL14, according to the Quantum Theory of Atoms in Molecules (QTAIM). ${ }^{23-25}$

2.3. Intermolecular Interactions. Intermolecular interaction energies were computed from the optimized P-DFT structure (see Section 2.2), considering all the symmetry-independent molecular pairs with a center-of-mass distance less than $20 \AA$. Single-point DFT (S-DFT) calculations were carried out on each pair extracted from the crystal at the dispersion-corrected $m$-GGA B3LYP/pob-TZVP ${ }^{26}$ level of theory using the Gaussian09 program package. ${ }^{27}$ All the pair energies were corrected for basis set superposition error (BSSE) by the counterpoise method ${ }^{28}$ and for relaxation energy with respect to the optimized monomers in the gas phase. ${ }^{29-32}$ Intermolecular interaction energies were also evaluated by the Spackman's experimental charge density approach (ECDA $)^{33-37}$ through the software PAMoC. ${ }^{88}$ Within this model, atom-atom partitioning allows us to single out estimates for individual hydrogen bond (HB) energies. For the sake of comparison, the latter were also estimated through the natural bond order (NBO) analysis of the wave function. ${ }^{39}$ From the ED topology
Table 1. Crystallographic Details and Refinement Statistics of the Title Compound $\left(\mathrm{C}_{18} \mathrm{H}_{28} \mathrm{~N}_{3} \mathrm{Cl}^{2+}\left(\mathrm{H}_{2} \mathrm{PO}_{4}^{-}\right)_{2} \cdot 2 \mathrm{H}_{2} \mathrm{O}\right)^{a}$

$\begin{array}{lll}T[\mathrm{~K}] & \mathrm{RT} & 103(2) \\ a[\AA]^{b} & 9.8350(2) & 9.7212(1) \\ b[\AA]^{b} & 16.8654(3) & 16.7733(2) \\ c[\AA]^{b} & 15.7859(3) & 15.6966(2) \\ \beta[\mathrm{deg}]^{b} & 105.750(1) & 105.1788(2) \\ V\left[\AA^{3}\right]^{b} & 2520.12(8) & 2470.14(5) \\ D_{\mathrm{x}}\left[\mathrm{g} \mathrm{cm}^{-3}\right] & 1.455 & 1.484 \\ \lambda[\AA], \mu\left[\mathrm{mm}^{-1}\right] & 0.71073,0.335 & 0.71073,0.342 \\ \text { crystal size }\left[\mathrm{mm}^{3}\right] & 0.725,0.620, & 0.725,0.620,0.425 \\ & 0.425 & \\ \text { reflns collected, unique, unique } & 45524,8218,7164 & 236057,20697, \\ >2 \sigma(I) & & 18642 \\ \text { completeness }(\%) & 98.6 & 100.0 \\ (\sin \theta / \lambda)_{\max }\left[\AA^{-1}\right] & 0.72 & 1.0 \\ R_{\text {int }} & 0.0155 & 0.0277\end{array}$

$R_{\text {int }}$

0.0155

0.0277

Refinement (Shelx)

refined parameters, data-to-

parameter ratio

$427,18.04$

$381,54.32$

$R_{\mathrm{F}}{ }^{2}, w R_{\mathrm{F}}^{2}$, goodness-of-fit (all data) $0.0438,0.1138, \quad 0.0427,0.1294$,

$\begin{array}{lcc} & 1.056 & 1.154 \\ \rho_{\mathrm{MAX} / \mathrm{MIN}}\left[\mathrm{e} \AA^{-3}\right]^{c} & +0.91,-0.26 & +2.00,-0.89\end{array}$

${ }^{a}$ Monoclinic, $P 2_{1} / c, F_{000}=1168$ e, $M_{\mathrm{w}}=551.89 \mathrm{amu}, Z=4$ and $Z^{\prime}=1$. $Z$ : space group multiplicity; $Z^{\prime}$ number of formula units per asymmetric unit (ASU), considering the whole ASU $\left(\mathrm{C}_{18} \mathrm{H}_{28} \mathrm{~N}_{3} \mathrm{Cl}^{2+}\right.$ $\left.\left(\mathrm{H}_{2} \mathrm{PO}_{4}^{-}\right)_{2} \cdot 2 \mathrm{H}_{2} \mathrm{O}\right)$ as one formula unit. In this structure, the ASU contains one diprotonated chloroquine molecule, $\mathrm{CQH}_{2}{ }^{2+}$, plus two symmetry-independent water molecules and two dihydrogen phosphate ions. ${ }^{b}$ Lattice constants at $103 \mathrm{~K}$ were obtained by the leastsquares fitting of the crystal orientation matrix against 29733 intense reflections integrated among $4.8^{\circ} \leq 2 \theta \leq 107.8^{\circ}$. At RT, the same quantities are 9193 and $4.4^{\circ} \leq 2 \theta \leq 60.9^{\circ}$. ${ }^{c} \mathrm{High}$ Fourier residuals at RT and $T=103(2) \mathrm{K}$ are partly due to disorder affecting water molecules. See Section 3.1 below.

viewpoint, PAMoC was employed to extract QTAIM descriptors from the S-DFT ED, complementing the in-crystal results provided by TOPOND. NCIs were studied also through visual inspection of isosurfaces of the reduced density gradient (RDG) descriptor, ${ }^{40,41}$ computed by the "NCImilano" code, ${ }^{42}$ according to the same procedure described elsewhere. ${ }^{41}$ The step size of the grids was set at $0.1 \mathrm{au}$, and RDG was calculated only for those molecular regions representative of intermolecular interactions, namely, with $0.00<\rho(\mathbf{r})$ $<0.05$ au. The $\rho(\mathbf{r}) \cdot \operatorname{sign}\left(\lambda_{2}\right)$ quantity, $\lambda_{2}$ being the second largest eigenvalue of the ED Hessian matrix at $\mathbf{r}$, was plotted on RDG isosurfaces with an isovalue of 0.4 if not otherwise specified.

The different recipes here employed enable to highlight different aspects of the recognition process of $\mathrm{CQ}$. NBO estimates compare $\mathrm{H}$ bonds on a common energy scale, while empirical ECDA terms provide a reasonable energy decomposition scheme into electrostatic, dispersion, and Pauli repulsion contributions and DFT interaction energies account for the whole energy balance.

\section{RESULTS AND DISCUSSION}

3.1. Crystal Structure of Chloroquine Hydrogenophosphate at $103 \mathrm{~K}$. The crystal structure of the $\mathrm{CQH}_{2}{ }^{2+}$. $\left(\mathrm{H}_{2} \mathrm{PO}_{4}^{-}\right)_{2} \cdot 2 \mathrm{H}_{2} \mathrm{O}$ salt was first solved by Preston \& Stewart in $1970^{43}$ and then more accurately redetermined in late 1980s by Karle \& Karle $^{44}$ (hereinafter, KK; CSD refcode: GEXXAI). The present experiments, conducted up to an unprecedented resolution and accuracy, confirm the main structural features previously described, but at variance with $\mathrm{KK}$ evidence of disorder involving $\mathrm{H}_{2} \mathrm{PO}_{4}{ }^{-}$ions and water molecules was found (see Section S2, Supporting Information for a detailed discussion). Fully protonated CQ crystallizes in the monoclinic 
$P 2_{1} / c$ lattice, with one $\mathrm{CQH}_{2}{ }^{2+}$, two $\mathrm{H}_{2} \mathrm{PO}_{4}^{-}$ions, and two water molecules in the asymmetric unit (ASU, Figure 1). The

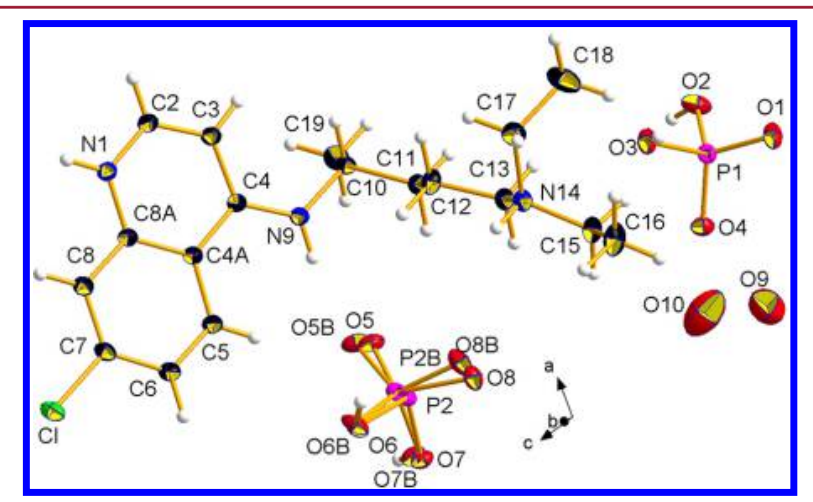

Figure 1. Asymmetric unit (ASU) of the title compound at $T=103(2)$ $\mathrm{K}$, with atoms numbering scheme. Thermal ellipsoids are drawn at the $50 \%$ probability level. Standard atomic color codes were employed for different elements (C: black; $\mathrm{H}$ : white; $\mathrm{O}$ : red; $\mathrm{N}$ : blue; $\mathrm{P}$ : purple, $\mathrm{Cl}$ : green). All the molecular pictures in this work were realized with the Diamond package (Diamond - Crystal and Molecular Structure Visualization. Crystal Impact - Dr. H. Putz \& Dr. K. Brandenburg GbR, Kreuzherrenstrasse 102, 53227 Bonn, Germany, http://www. crystalimpact.com/diamond).

hydrocarbon side chains in each pair of $2_{1}$-related drug molecules wrap around a single phosphate pillar, setting up a sort of helical arrangement (Figure 2a) reinforced by strong CAHBs interactions (Table S2, Supporting Information). Just weak $\mathrm{CH} \cdots \mathrm{O}$ contacts, deemed not significant for the overall structure stability, ${ }^{45}$ are set up among $\mathrm{CQH}_{2}{ }^{2+}$ and water (see also Section 3.2 and Section S2.3, Supporting Information). As expected, $\mathrm{CQH}_{2}{ }^{2+}$ shows a neat preference toward direct interactions with the $\mathrm{H}_{2} \mathrm{PO}_{4}{ }^{-}$counterions (Table S2, Supporting Information). In each unit cell, dihydrogen phosphate ions form two symmetry-related infinite chains parallel to the monoclinic $b$ axis (Figure 2a), while the quinoline system of $\mathrm{CQH}_{2}{ }^{2+}$ roughly lies in the orthogonal ( $a$, c) plane (Figure $2 \mathrm{~b}$ ). Each $\mathrm{P}_{1} \mathrm{H}_{2} \mathrm{PO}_{4}{ }^{-}$ion accepts a couple of strong CAHBs involving both the $\mathrm{N} 1$ and $\mathrm{N} 14$ charged $-\mathrm{NH}^{+}$ groups from two distinct chloroquine molecules (Table S2, Supporting Information). On the contrary, the P2 ion is involved just in a weaker $\mathrm{NH} \cdots \mathrm{O}$ interaction with the uncharged N9-H amine (Table S2, Supporting Information).
This could be related to the persistence of residual dynamic disorder down to $\sim 100 \mathrm{~K}$ (see Section S2, Supporting Information) for this ion.

Finally, it is worth mentioning that pairs of inversion-related $\mathrm{H}_{2} \mathrm{PO}_{4}^{-}$pillars in adjacent unit cells are bound by a threedimensional zigzag motif of hydrogen-bonded bridging water molecules (Figure S1a, Supporting Information). According with $\mathrm{KK},{ }^{44}$ we were also not able to unequivocally locate hydrogen atoms around the two water oxygen atoms, $\mathrm{O} 9$ and O10, neither at RT nor at $103 \mathrm{~K}$. This is a sign of another kind of disorder, which in this case intrinsically concerns the $\mathrm{H}$ atoms of the water molecules due to $\mathrm{HB}$ frustration. Further details can be found in the Section S2 of the Supporting Information.

3.2. A Quantum Model for the Chloroquine Dihydrogen Phosphate Dihydrate Salt. This work aims at recognizing what are the relevant intermolecular NCI accounting for the peculiar molecular recognition features of chloroquine. Assuming that these latter are governed by intrinsic and stable ED properties (electrophilicity, nucleophilicity, electrostatics, ability of setting up hydrogen bonds...), we hypothesize that the crystal structure should qualitatively mimic the essential aspects of the drug-substrate recognition mode. ${ }^{46-48}$ In this respect, it should be noted that the action of $\mathrm{CQ}$ does not directly involve any metabolic process and relies on purely local chemical factors, such as the $\mathrm{pH}$ and the presence of surfactants. ${ }^{12}$ Therefore, we first study the crystal structure of the $\mathrm{CQH}_{2}{ }^{2+}$ dication, which is the prevailing form in the acidic DV of Plasmodium. The simultaneous occurrence of disorder, diffuse scattering, and frustration-forced symmetry lowering (see Section S2.1, Supporting Information) hampered us from obtaining a reliable experimental ED at $T=103 \mathrm{~K}$. We thus resorted to a quantum all-electron LCGTF P-DFT model for the in-crystal charge density (Section 2.2). Since the water molecules have just a minor influence on the crystal structure and on the CQ conformation, as demonstrated in Sections S2.4 and S3, Supporting Information, the full $P 2_{1} / c$ lattice symmetry was exploited in P-DFT simulations, implying that the frustrated $\mathrm{H}_{2} \mathrm{O}$-chain motif between dihydrogen phosphate pillars is not conserved (Section S2.4, Supporting Information). Attractive interactions between oppositely charged groups are known to possibly dominate the heme/CQ recognition process. $^{32}$ Accordingly, dihydrogen phosphate ions should represent a suitable model for interactions with the propionate

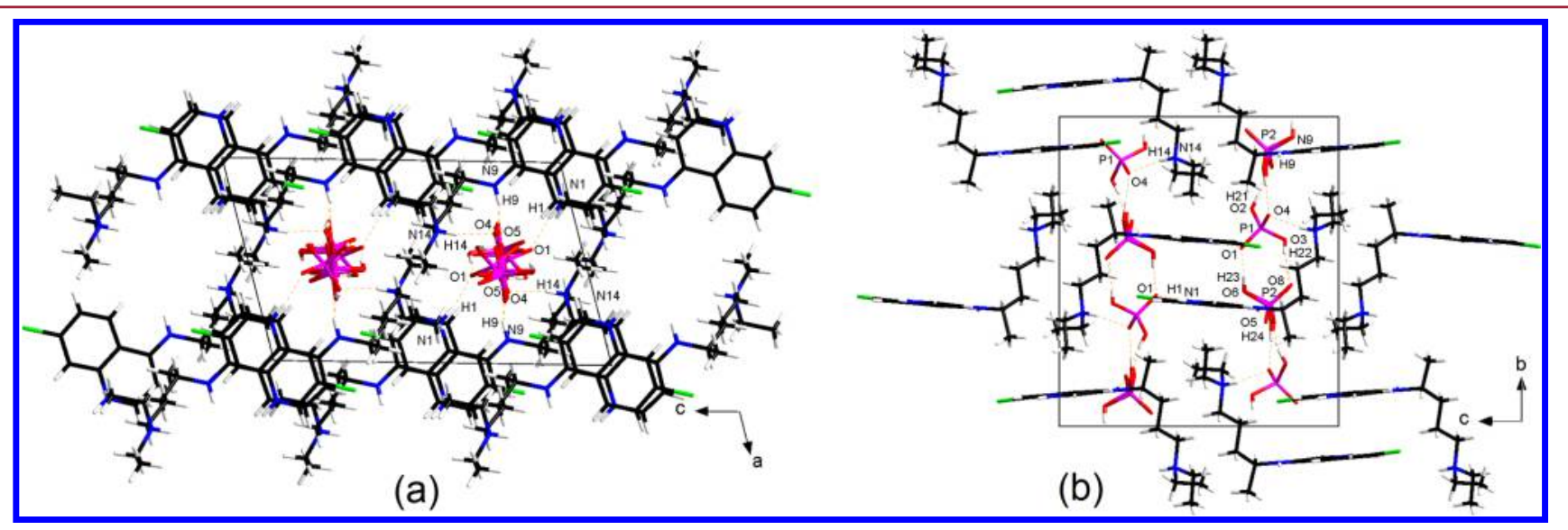

Figure 2. Wire-and-stick representation of the crystal packing of the title compound at $T=103(2) \mathrm{K}$. Relevant $\mathrm{NH} \cdots \mathrm{O}$ and $\mathrm{OH} \cdots \mathrm{O}$ HBs $(\mathrm{Table} \mathrm{S} 2$, Supporting Information) are highlighted as red dotted lines. (a) View along the $b$ axis; (b) view along the $a$ axis. 
groups of heme in solution, ${ }^{12}$ while the stacking interactions between adjacent $\mathrm{CQH}_{2}{ }^{2+}$ molecules along $b$ (Figure 2) might serve as a probe for the ability of the drug to similarly approach the flat protoporphyrin ring.

3.2.1. ED-Based Analysis of In-Crystal Intermolecular Interactions. 3.2.1.1. Chloroquine-Dihydrogen Phosphate. $\mathrm{CQH}_{2}{ }^{2+} \ldots \mathrm{H}_{2} \mathrm{PO}_{4}{ }^{-}$interactions are invariably stabilizing due to strong Coulombic attraction between opposite charges. Figure 3 shows the interaction geometries of some most attractive

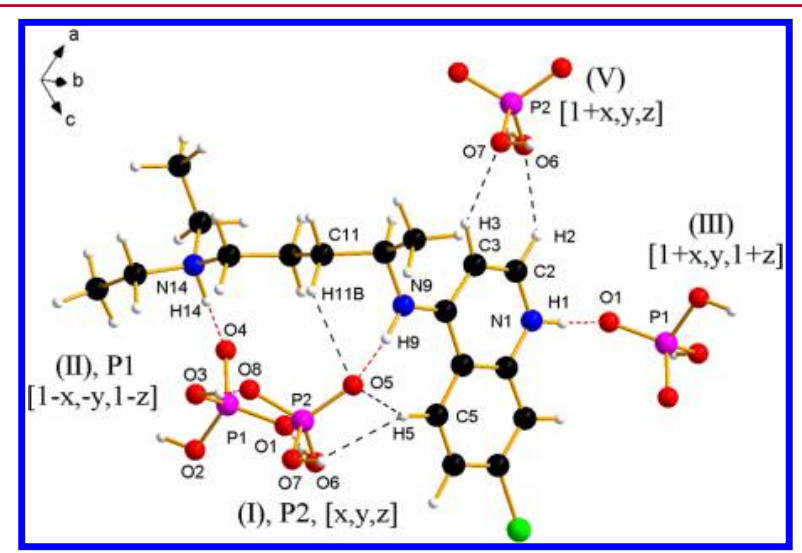

Figure 3. Most attractive $\mathrm{CQH}_{2}{ }^{2+} \ldots \mathrm{H}_{2} \mathrm{PO}_{4}{ }^{-}$molecular pairs in the first coordination shell of chloroquine, i.e., with distance between centers of mass, $d$, less than $8 \AA$. Roman numerals label pairs in descending order of $m$-GGA DFT interaction energies (see Section 3.2.2). Contacts corresponding to relevant QTAIM AILs are shown as dashed lines, with the following color code: $\mathrm{OH} \cdots \mathrm{O}, \mathrm{NH} \cdots \mathrm{O}$ in red; $\mathrm{CH} \cdots \mathrm{O}$ in black. Symmetry operations are also indicated. The atomic colors are the same as in Figure 1.

$\mathrm{CQH}_{2}{ }^{2+} \cdots \mathrm{H}_{2} \mathrm{PO}_{4}^{-}$pairs in the first coordination shell of chloroquine which exploit relevant intermolecular hydrogen bonded contacts, as summarized in Table 2. By relevant we mean those contacts for which an atomic interaction line (AIL) exists with charge density at the bond critical point, $\rho_{\mathrm{bcp}}>10^{-2}$ au. The interested reader can find more details on this topic, including a complete list of atom-atom contacts, in Section S3, Supporting Information. If not otherwise specified, the QTAIM investigation was always performed on the ED of molecular pairs extracted from the crystal (Section 2.3). However, point topological properties in the solid state are in very good agreement with those evaluated in vacuo (Tables S6-S7, Supporting Information). Both geometry and QTAIM point properties (Table 2 ) confirm that $\mathrm{NH} \cdots \mathrm{O}$ HBs are significantly stronger than $\mathrm{CH} \cdots \mathrm{O}$ ones. $\mathrm{NH} \cdots \mathrm{O}$ distances $(1.6-1.9 \AA)$ are much shorter (up to $40 \%$ ) than the sum of $\mathrm{H}$ and $\mathrm{O}$ standard van der Waals radii (e.g., $2.7 \AA$ according to Bondi ${ }^{49}$ ). The closest $\mathrm{H} 1 \cdots \mathrm{O} 1$ and $\mathrm{H} 14 \cdots \mathrm{O} 4$ contacts are also strongly charge-assisted, as they involve direct interactions among protonated amine nitrogens and partly negative $\mathrm{O}^{-}$atoms in the P1 dihydrogen phosphate ion.

Accordingly, such contacts show high $\rho_{\mathrm{bcp}}$ and negative local energy density $H_{\mathrm{bcp}}\left(H_{\mathrm{bcp}}=G_{\mathrm{bcp}}+V_{\mathrm{bcp}}\right)$ values, at variance with all the other contacts examined in Table 2 . Negative $H_{\text {bcp }} / \rho_{\text {bcp }}$ ratios are usually associated with the incipient formation of covalent bonds ${ }^{50}$ and can be so considered as a symptom of partial $\mathrm{H} \cdots \mathrm{O}$ covalency.

The secondary amine substituent of the quinoline ring, $\mathrm{N} 9 \mathrm{H} 9$, is involved in the third CAHB with $\mathrm{O} 5$ in the $\mathrm{P} 2$ $\mathrm{H}_{2} \mathrm{PO}_{4}{ }^{-}$ion, but this bond is somewhat weaker than the other two, the $\mathrm{NH}$ group being formally neutral. Its bcp electron density is about only 3 times higher than that of $\mathrm{CH} \cdot \cdots \mathrm{O}$ bonds and has a marginally positive energy density (Table 2).

A very good agreement emerges between the QTAIM picture and that based on the RDG. As expected, ${ }^{40,41}$ strong $\mathrm{NH}$... O HBs are invariably associated with contracted, discshaped RDG isosurfaces with $\operatorname{sign}\left(\lambda_{2}\right) \cdot \rho(\mathbf{r}) \ll 0$ (Figure 4$)$. On the contrary, $\mathrm{CH} \cdots \mathrm{O}$ interactions reflect in larger and more structured isosurfaces dominated by slightly negative or neutral $\lambda_{2}$ curvatures, sometimes enclosing both the $\mathrm{H} \cdots \mathrm{O}$ bcp and the ring critical point (see for example pairs (I) and (III) in Figure 4) formed by the $\mathrm{CH} \cdots \mathrm{O}$ interaction. It is also worth noting that some very weak intramolecular $\mathrm{H} \cdots \mathrm{H}$ contacts, characterized by $\operatorname{sign}\left(\lambda_{2}\right) \cdot \rho \sim 0$ zones adjacent to $\operatorname{sign}\left(\lambda_{2}\right) \cdot \rho>0$ surfaces, are evident in the RDG plots, regardless of whether the corresponding bcp is present or not in the ED distribution of the isolated pair (for example, consider the $\mathrm{H} 3 \cdots \mathrm{H} 10$ and H5 $\cdots \mathrm{H} 9$ contacts in pair (III), Figure 4 and Table S6, Supporting Information). These features reflect the presence of rather flat regions of the charge density, where $|\nabla \rho|$ approaches 0 or where the RDG has just a minimum. The latter regions are very common ${ }^{41}$ for very weak closed-shell

Table 2. Bond Path Lengths, $d_{\mathrm{H} \cdots \mathrm{A}}$ and Topological Properties at the Bond Critical Points (bcp's) of Relevant Noncovalent Bonded Contacts, as shown in Figure $3^{a}$

\begin{tabular}{|c|c|c|c|c|c|c|}
\hline $\operatorname{contact}^{b}$ & $d_{\mathrm{H} \cdots \mathrm{A}} / \AA$ & $\rho_{\mathrm{bcp}} / \mathrm{au}$ & $\nabla^{2} \rho_{\mathrm{bcp}} / \mathrm{au}$ & $G_{\mathrm{bcp}} / \mathrm{kcal} \cdot \mathrm{mol}^{-1} \cdot \AA^{-3}$ & $V_{\mathrm{bcp}} / \mathrm{kcal} \cdot \mathrm{mol}^{-1} \cdot \AA^{-3}$ & $\mathrm{BD}^{c} / \mathrm{au}$ \\
\hline \multicolumn{7}{|c|}{ Pair I, $\mathrm{CQH}_{2}{ }^{2+} \ldots \mathrm{H}_{2} \mathrm{P}_{2} \mathrm{O}_{4}^{-}[\mathrm{E}]$} \\
\hline N9-H9”OO5 & 1.8674 & 0.031 & 0.102 & 15.6 & -15.2 & 0.02 \\
\hline $\mathrm{C} 5-\mathrm{H} 5 \cdots \mathrm{O} 5$ & 2.4455 & 0.011 & 0.037 & 4.8 & -3.9 & 0.13 \\
\hline $\mathrm{C} 5-\mathrm{H} 5 \cdots \mathrm{O} 6$ & 2.4824 & 0.010 & 0.034 & 4.5 & -3.6 & 0.15 \\
\hline $\mathrm{C} 11-\mathrm{H} 11 \mathrm{~B} \cdots \mathrm{O} 5$ & 2.5569 & 0.009 & 0.031 & 4.1 & -3.2 & 0.14 \\
\hline \multicolumn{7}{|c|}{ Pair II, $\mathrm{CQH}_{2}{ }^{2+} \ldots \mathrm{H}_{2} \mathrm{P} \mathrm{O}_{4}^{-}[\mathrm{i}]$} \\
\hline 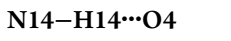 & 1.7419 & 0.045 & 0.115 & 22.1 & -26.1 & -0.14 \\
\hline \multicolumn{7}{|c|}{ Pair III, $\mathrm{CQH}_{2}{ }^{2+} \ldots \mathrm{H}_{2} \mathrm{P} \mathrm{O}_{4}^{-}[\mathrm{E}]$} \\
\hline $\mathrm{N} 1-\mathrm{H} 1 \cdots \mathrm{O} 1$ & 1.6279 & 0.056 & 0.129 & 27.5 & -34.8 & -0.21 \\
\hline \multicolumn{7}{|c|}{ Pair $\mathrm{V}, \mathrm{CQH}_{2}{ }^{2+} \ldots \mathrm{H}_{2} \mathrm{P}_{2} \mathrm{O}_{4}{ }^{-}[\mathrm{E}]$} \\
\hline $\mathrm{C} 2-\mathrm{H} 2 \cdots \mathrm{O} 6$ & 2.2487 & 0.015 & 0.057 & 7.2 & -5.5 & 0.18 \\
\hline $\mathrm{C} 3-\mathrm{H} 3 \cdots \mathrm{O} 7$ & 2.2700 & 0.014 & 0.053 & 6.8 & -5.2 & 0.17 \\
\hline
\end{tabular}

${ }^{a}$ CAHBs interactions are highlighted in bold. $G_{\mathrm{bcp}}$ and $V_{\mathrm{bcp}}$ are the electronic kinetic and potential energy densities, as computed from the wave function. ${ }^{b}$ Crystal point symmetries involved in the construction of the pair are given in square brackets. ${ }^{c}$ Bond degree (BD) parameter, according with Espinosa and co-workers, defined as $H_{\mathrm{bcp}} / \rho_{\mathrm{bcp}} .{ }^{50}$ 


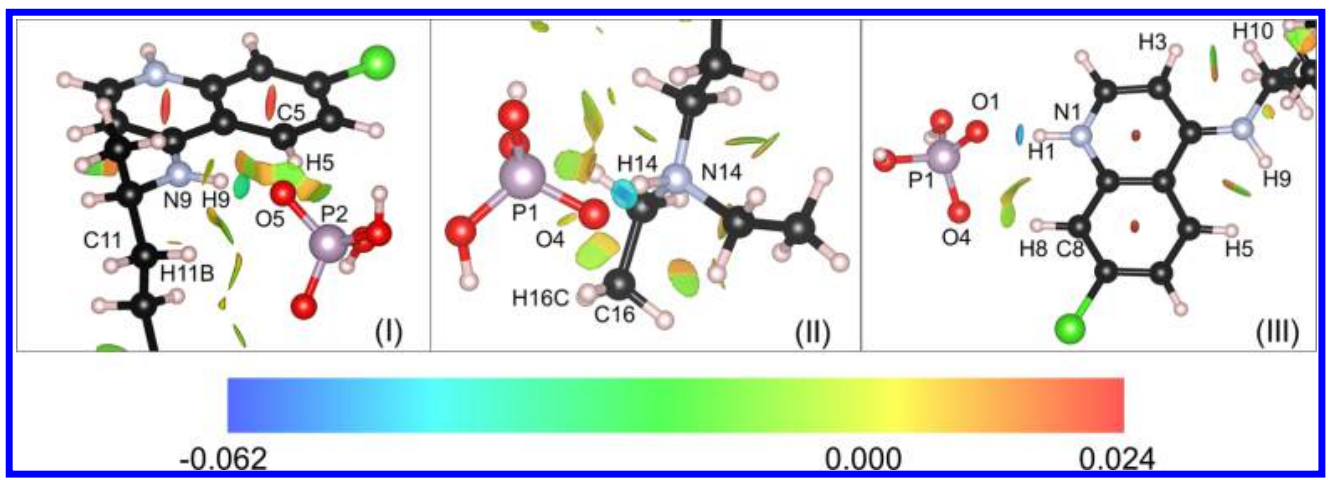

Figure 4. $\operatorname{Sign}\left(\lambda_{2}\right) \cdot \rho$ function plotted on RDG isosurfaces in the internuclear region of the most attractive molecular pairs (I-III). Values on the chromatic scale are given in atomic units.

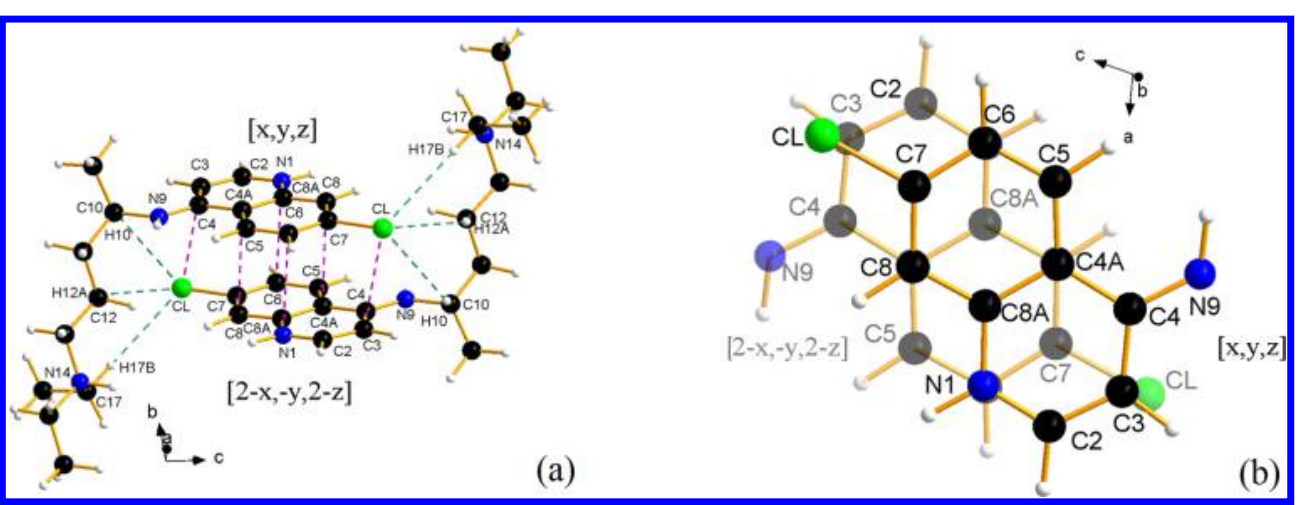

Figure 5. (a) Most repulsive $\mathrm{CQH}_{2}{ }^{2+} \ldots \mathrm{CQH}_{2}{ }^{2+}$ pair $\left(d=5.57 \AA\right.$, $E_{\text {int,DFT }}=+153.5 \mathrm{kcal} \cdot \mathrm{mol}^{-1}$, see Figure $S 7$ and Table S8, Supporting Information $)$ in the title compound at $T=103 \mathrm{~K}$. Contacts corresponding to relevant QTAIM AILs are shown as dashed lines, with the following color code: $\mathrm{CH} \cdots \mathrm{Cl}$ in green; $\mathrm{C} \cdots \mathrm{X}, \mathrm{X}=\mathrm{C}, \mathrm{N}, \mathrm{Cl}$ in purple. (b) Same as (a), with focus on the region of the quinoline system viewed roughly down the $\mathrm{C} \cdots \mathrm{X}$ AILs. Nuanced atoms lie below the plane of the figure.

Table 3. Bond Path Lengths, $d_{\mathrm{X} \cdots \mathrm{A}}$ and Topological Parameters at the Bond Critical Points (bcp's) of Relevant Noncovalent Contacts shown in Figure $5^{a}$

\begin{tabular}{|c|c|c|c|c|c|c|}
\hline contact & $d_{\mathrm{X} \cdots \mathrm{A}} / \AA$ & $\rho_{\mathrm{bcp}} / \mathrm{au}$ & $\nabla^{2} \rho_{\mathrm{bcp}} / \mathrm{au}$ & $G_{\mathrm{bcp}} / \mathrm{kcal} \cdot \mathrm{mol}^{-1} \cdot \AA^{-3}$ & $V_{\mathrm{bcp}} / \mathrm{kcal} \cdot \mathrm{mol}^{-1} \cdot \AA^{-3}$ & $\mathrm{BD} / \mathrm{au}$ \\
\hline \multicolumn{7}{|c|}{$\mathrm{CQH}_{2}{ }^{2+} \ldots \mathrm{CQH}_{2}{ }^{2+}[\mathrm{i}]$} \\
\hline $\mathrm{C} 3 \cdots \mathrm{Cl}$ & 3.4497 & 0.007 & 0.022 & 3.0 & -2.4 & 0.13 \\
\hline $\mathrm{C} 6 \cdots \mathrm{N} 1$ & 3.4857 & 0.005 & 0.021 & 2.4 & -1.6 & 0.25 \\
\hline $\mathrm{C} 4 \mathrm{~A} \cdots \mathrm{C} 8$ & 3.5204 & 0.004 & 0.019 & 2.1 & -1.2 & 0.32 \\
\hline $\mathrm{C} 12-\mathrm{H} 12 \mathrm{~A} \cdots \mathrm{Cl}$ & 3.0079 & 0.007 & 0.021 & 2.7 & -2.2 & 0.12 \\
\hline $\mathrm{C} 17-\mathrm{H} 17 \mathrm{~B} \cdots \mathrm{Cl}$ & 3.2650 & 0.003 & 0.010 & 1.3 & -1.0 & 0.13 \\
\hline $\mathrm{C} 10-\mathrm{H} 10 \cdots \mathrm{Cl}$ & 3.3902 & 0.003 & 0.011 & 1.3 & -0.9 & 0.23 \\
\hline
\end{tabular}

${ }^{a}$ See Table 2 for the meaning of the various quantities.

interactions, lacking a QTAIM AIL, ${ }^{51}$ and they can be hardly deemed as significant in determining the observed in-crystal folding of the drug.

3.2.1.2. Chloroquine Self-Recognition. The most repulsive $\mathrm{CQH}_{2}{ }^{2+} \ldots \mathrm{CQH}_{2}{ }^{2+}$ pair (Figure S7 and Table S8, Supporting Information) involves inversion-related $\pi$-stacked chloroquine

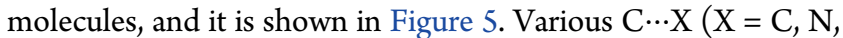
$\mathrm{Cl}$ ) and $\mathrm{CH} \cdots \mathrm{Cl}$ interactions, each associated with a topological AIL (Table 3), are present in this pair. They are characterized by 1 order of magnitude lower $\rho_{\text {bcp }}$ values than for the $\mathrm{NH} \cdots \mathrm{O}$ and $\mathrm{CH} \cdots \mathrm{O}$ bonds shown in Table 2 . However, while $\mathrm{CH} \cdots \mathrm{Cl}$ contacts show topological parameters very similar to those of the weakest $\mathrm{CH} \cdots \mathrm{O}$ interactions (Table S6, Supporting Information), the $\mathrm{C} \cdots \mathrm{X}$ ones are systematically a bit stronger in terms of $\rho_{\mathrm{bcp}}$ and $V_{\mathrm{b}}$ estimates. From a geometrical viewpoint, the two flat rings are head-to-tail packed (see
Section 3.1), with a least-squares distance between the condensed quinoline moieties as high as $3.37 \AA$. For the sake of comparison, the van der Waals contact distance between $\mathrm{C}$ atoms is $\sim 3.4 \AA^{49}$ Interestingly, $\mathrm{C} \cdots \mathrm{X}$ AILs, i.e., C3 $\cdots \mathrm{Cl}, \mathrm{C} 6 \cdots$ $\mathrm{N} 1$, and $\mathrm{C} 4 \mathrm{~A} \cdots \mathrm{C} 8$, are established between pairs of atoms mutually displaced along the $\mathrm{C} 3-\mathrm{C} 8 \mathrm{~A}$ ring diagonal, so that atoms $\mathrm{C} 7$ and $\mathrm{C} 8 \mathrm{~A}$ are reciprocally positioned roughly at the geometrical center of facing six-membered rings (Figure 5b). Such stacking motifs are very common in systems containing terminal flat aromatic rings bonded to somewhat long hydrocarbon chains, such as in $\mathrm{DNA}^{52}$ and DNA-protein complexes. ${ }^{53}$ Both recently and in the past they were deemed as a possible signature of $\pi \cdots \pi$ interactions, ${ }^{52-54}$ while the properties of cage critical points (ccp's) emerging from cyclic AIL patterns in the stacking region were correlated to 


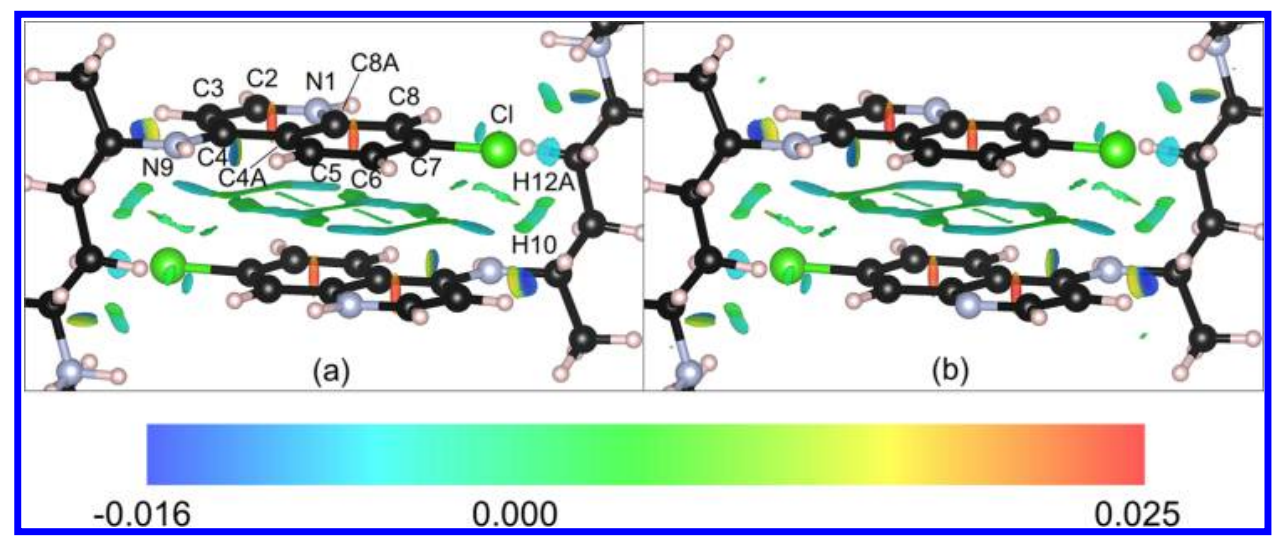

Figure 6. (a) $\operatorname{Sign}\left(\lambda_{2}\right) \cdot \rho$ function plotted on RDG isosurfaces in the internuclear region of the most repulsive molecular pair $\mathrm{CQH}_{2}{ }^{2+} \ldots \mathrm{CQH}_{2}{ }^{2+}$. (b) Same as (a), for a model pair of inversion-related neutral chloroquine molecules placed at the same positions occupied by their protonated counterparts in the crystal. Values on the chromatic scale are given in atomic units.

Table 4. Interaction Energies Vs. the Center of Mass Distance $d$ of the Most Attractive and Repulsive Molecular Pairs (Figures 3 and 5) in the First Coordination Shell of Chloroquine in the Title Compound ${ }^{a}$

\begin{tabular}{|c|c|c|c|c|c|c|c|c|c|}
\hline pair & $d$ & $E_{\mathrm{DFT}}^{b}$ & $E_{\mathrm{ECDA}}{ }^{c}$ & $E_{\mathrm{r}}^{c}$ & $E_{\mathrm{d}}^{c}$ & $E_{\mathrm{es}}{ }^{c}$ & hydrogen bonds & $E(2)^{d}$ & $E_{\mathrm{HB}}{ }^{d}$ \\
\hline \multirow[t]{4}{*}{$\mathrm{CQH}_{2}{ }^{2+} \ldots \mathrm{H}_{2} \mathrm{PO}_{4}^{-}(\mathrm{I})^{e}$} & 4.11 & -133.7 & -127.4 & 20.0 & -7.0 & -140.4 & $\mathrm{~N} 9-\mathrm{H} 9 \cdots \mathrm{O} 5$ & 19.3 & 11.9 \\
\hline & & & & & & & $\mathrm{C} 5-\mathrm{H} 5 \cdots \mathrm{O} 5$ & 1.9 & 1.5 \\
\hline & & & & & & & $\mathrm{C} 5-\mathrm{H} 5 \cdots \mathrm{O} 6$ & 1.3 & 1.3 \\
\hline & & & & & & & $\mathrm{C} 11-\mathrm{H} 11 \mathrm{~B} \cdots \mathrm{O} 5$ & 1.2 & 1.0 \\
\hline $\mathrm{CQH}_{2}{ }^{2+} \cdots \mathrm{H}_{2} \mathrm{PO}_{4}^{-}(\mathrm{II})^{e}$ & 4.25 & -133.3 & -139.1 & 27.1 & -7.9 & -158.3 & $\mathrm{~N} 14-\mathrm{H} 14 \cdots \mathrm{O} 4$ & 33.4 & 18.4 \\
\hline $\mathrm{CQH}_{2}{ }^{2+} \ldots \mathrm{H}_{2} \mathrm{PO}_{4}^{-}(\mathrm{III})^{e}$ & 7.66 & -108.7 & -106.4 & 29.8 & -4.8 & -131.4 & $\mathrm{~N} 1-\mathrm{H} 1 \cdots \mathrm{O} 1$ & 46.9 & 27.9 \\
\hline \multirow[t]{2}{*}{$\mathrm{CQH}_{2}{ }^{2+} \ldots \mathrm{H}_{2} \mathrm{PO}_{4}^{-}(\mathrm{V})^{e}$} & 6.30 & -83.5 & -94.6 & 8.4 & -3.8 & -99.1 & $\mathrm{C} 2-\mathrm{H} 2 \cdots \mathrm{O} 6$ & 3.8 & 3.2 \\
\hline & & & & & & & $\mathrm{C} 3-\mathrm{H} 3 \cdots \mathrm{O} 7$ & 4.2 & 2.8 \\
\hline \multirow[t]{3}{*}{$\mathrm{CQH}_{2}^{2+} \ldots \mathrm{CQH}_{2}^{2+}$} & 5.57 & 153.5 & 136.9 & 19.9 & -19.4 & 136.3 & $\mathrm{C} 12-\mathrm{H} 12 \mathrm{~A} \cdots \mathrm{Cl}$ & 0.5 & 0.7 \\
\hline & & & & & & & $\mathrm{C} 17-\mathrm{H} 17 \mathrm{~B} \cdots \mathrm{Cl}$ & 0.4 & 0.2 \\
\hline & & & & & & & $\mathrm{C} 10-\mathrm{H} 10 \cdots \mathrm{Cl}$ & 0.1 & 0.1 \\
\hline \multirow[t]{3}{*}{$\mathrm{CQ} \cdots \mathrm{CQ}^{f}$} & 5.57 & 9.7 & -11.6 & 19.5 & -18.7 & -12.4 & $\mathrm{C} 12-\mathrm{H} 12 \mathrm{~A} \cdots \mathrm{Cl}$ & 0.4 & 0.7 \\
\hline & & & & & & & $\mathrm{C} 17-\mathrm{H} 17 \mathrm{~B} \cdots \mathrm{Cl}$ & 0.4 & 0.2 \\
\hline & & & & & & & $\mathrm{C} 10-\mathrm{H} 10 \cdots \mathrm{Cl}$ & 0.1 & 0.1 \\
\hline
\end{tabular}

${ }^{a}$ Values are given in $\AA$ and $\mathrm{kcal} \cdot \mathrm{mol}^{-1}$. ${ }^{b}$ BSSE-corrected $m$-GGA B3LYP pob-TZVP interaction energies for molecular pairs extracted from the crystal. ${ }^{c}$ Spackman's ECDA semiempirical interaction energies. ${ }^{33-37}{ }^{d}$ Hydrogen bond energies, intended as the energies required to break the $\mathrm{H} \cdots$ acceptor interactions, evaluated within the DFT and ECDA models. $E(2)$ comes from the second-order perturbative estimate of donor-acceptor interactions in the NBO basis according to $E(2)=q_{\mathrm{i}} \cdot F(i, j)^{2} /\left(\varepsilon_{\mathrm{i}}-\varepsilon_{\mathrm{j}}\right), q_{\mathrm{i}}$ being the donor orbital occupancy, $\varepsilon_{\mathrm{i}}-\varepsilon_{\mathrm{j}}$ the eigenvalue energy difference of the involved orbitals and $F(i, j)$ the corresponding off-diagonal NBO Kohn-Sham matrix element. $E_{\mathrm{HB}}$ derives from the semiempirical ECDA formalism as the sum of atom-atom $\mathrm{H} \cdots$ acceptor pairwise attractive terms. ${ }^{35,36} E_{\mathrm{r}}$ is locally ignored for such interactions to model the unique ability of $\mathrm{H}$ atoms to penetrate the van der Waals sphere of their acceptors, without arousing a significant exchange-repulsion contribution. ${ }^{e}$ Roman numerals rank the pairs toward less negative DFT energies. See Table S9, Supporting Information for a complete list. ${ }^{f}$ Interaction energies between two chloroquine neutral molecules kept exactly at the same positions of the most repulsive $\mathrm{CQH}_{2}{ }^{2+} \ldots \mathrm{CQH}_{2}{ }^{2+}$ charged pair.

stabilization energies of the interacting pairs ${ }^{54}$ (see Section 3.2.2 below).

Alternating AILs between the facing rings imply the presence of topological cages and the associated $(3,+3)$ cage critical points (ccps). This AIL motif implies that RDG isosurfaces also assume a strongly structured motif (Figure 6a), i.e., a bicyclic RDG pattern mirroring the shape of the quinoline backbone appears roughly halfway between the two chloroquine molecules. Interestingly, this surface is more flat and sharp than those usually associated with repulsive/dispersive interactions in other substances. ${ }^{41}$ The $\operatorname{sign}\left(\lambda_{2}\right) \cdot \rho(\mathbf{r})$ quantity, however, is invariably very low and often weakly positive, meaning that $\rho(\mathbf{r})$ in the internuclear region is also very low and with a $\lambda_{2}$ curvature close to zero-i.e., no local, directional ED accumulations, even incipient, due to specific noncovalent atom-atom interactions are present. In this respect, it is instructive to compare Figure $6 \mathrm{a}$ with Figure $6 \mathrm{~b}$, where the $\operatorname{sign}\left(\lambda_{2}\right) \cdot \rho(\mathbf{r})$ scalar is plotted onto the same $\mathrm{RDG}$ isosurface computed for a couple of neutral chloroquine molecules, kept exactly at the same positions their protonated counterparts have in the crystal. The above-described features are strictly conserved in the deprotonated molecular pair, together with the corresponding pattern of AILs (Tables S6-S7, Supporting Information). This implies that, within the adopted frozengeometry approximation, this specific RDG feature does not correlate with the acid-base status of chloroquine, as expected. Indeed, the electrostatic contribution plays here the major role, and it is known to be not directly reflected into point properties of the ED topology. ${ }^{55}$

3.2.2. Interaction Energetics. Analysis of interaction energies of molecular pairs at their in-crystal geometries highlights the relative weights of different NCI networks in determining the observed crystal structure, complementing the topological picture based on the ED features discussed in Section 3.2.1. Table 4 summarizes the DFT and semiempirical ECDA interaction energies of relevant pairs in the first 
coordination shell of $\mathrm{CQH}_{2}{ }^{2+}$, together with their decomposition into dispersive $\left(E_{\mathrm{d}}\right)$, repulsive $\left(E_{\mathrm{r}}\right)$, and electrostatic $\left(E_{\mathrm{es}}\right)$ contributions. The $\mathrm{HB}$ energies, as estimated through the ECDA model and the NBO analysis of the wave function, are also shown.

3.2.2.1. Coulomb-Driven Interactions and the Role of Hydrogen Bonds. The dominant role of pure Coulomb interactions between opposite charges as a true structurestabilizing factor can be appreciated in Figure 7, where $E_{\mathrm{DFT}}$ is

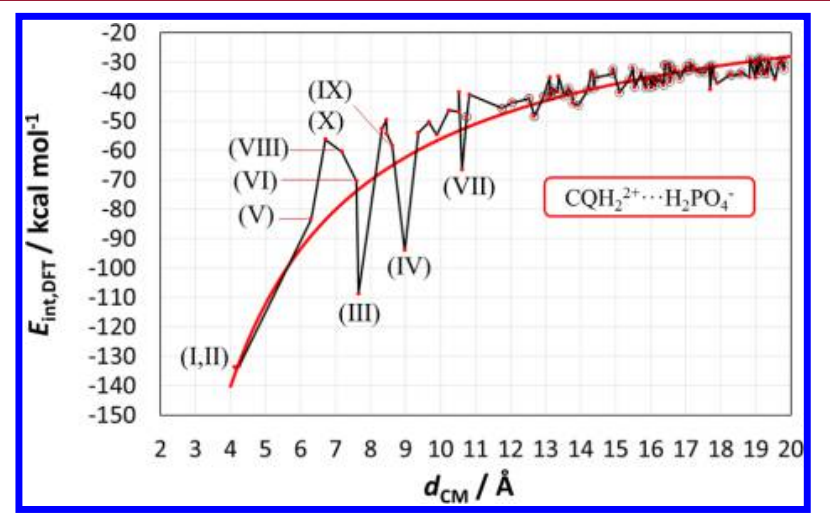

Figure 7. $m$-GGA B3LYP/pob-TZVP interaction energies, $E_{\mathrm{DFT}}$ (kcal. $\mathrm{mol}^{-1}$ ), for $\mathrm{CQH}_{2}{ }^{2+} \ldots \mathrm{H}_{2} \mathrm{PO}_{4}^{-}$pairs extracted from the DFT-relaxed crystal, as a function of the their center of mass distances, $d(\AA)$. The continuous red curve has equation $E_{\mathrm{LS}}(d)=-561(4) / d$ and comes from a Levenberg-Marquardt least-squares fit $(r=0.861,\langle\sigma\rangle=3$ $\mathrm{kcal} \cdot \mathrm{mol}^{-1}$ ) against the 85 points with $d>10 \AA$ enclosed in black circles, which lie within $2\langle\sigma\rangle$ from the corresponding predicted values. ${ }^{56}$ The black broken line serves as a guide for the eye. Topranking pairs are also marked with Roman numerals, in increasing order from most to less negative $E_{\mathrm{DFT}}$. Packing diagrams corresponding to pairs (I) $-(\mathrm{X})$ can be found in Figure 3 and in Figure S8, Supporting Information.

plotted for all the $\mathrm{CQH}_{2}{ }^{2+} . . \mathrm{H}_{2} \mathrm{PO}_{4}{ }^{-}$molecular pairs within $d=$ $20 \AA$. A full version of this graphic is available in the Supporting Information (Figure S7), together with the corresponding quantities in tabular form (Tables S8-S9, Supporting Information). At large center of mass separations, attractive interactions follow the expected $d^{-1}$ Coulomb law (curve $E_{\mathrm{LS}}$ in Figure 7) for the $+2 /-1$ charged chloroquine-phosphate pairs. Specific intermolecular contacts and/or aspherical features of the electrostatic potential, related to molecular extension and shape effects active at short distance, make some DFT interaction energy estimates significantly deviating from the $d^{-1}$ trend (Section S3.4, Supporting Information).

As expected, just the $\mathrm{NH} \cdots \mathrm{O}$ CAHBs are somewhat significant as possible structure determinants, as they account for a consistent part of the total interaction energy (Table 4). Their relative contributions follow the absolute strength of the

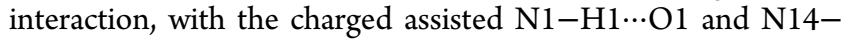

$\mathrm{H} 14 \cdots \mathrm{O} 4 \mathrm{HBs}$ providing $43.1 \%$ and $25.1 \%$ of the DFT energy and the neutral secondary amine N9-H9... O5 HB only $14.4 \%$ of such energy. Interestingly, the strongest $\mathrm{HB}$ on absolute grounds, $\mathrm{N} 1-\mathrm{H} 1 \cdots \mathrm{O} 1$, does not bridge the closer (and more stable) pairs (I) or (II), but the (III) one. This occurs frequently in organic crystals without strong HBs. ${ }^{45}$ The fact that it remains also true in the presence of CAHBs just confirms that there is not, in general, a 1:1 correspondence between very short atom-atom contacts and most stabilizing pairs. Rather, the picture is often more complex, and the interested reader can find further details in Section S3, Supporting Information.

3.2.2.2. Chloroquine-Chloroquine Interactions. The last rows in Table 4 show the energy contributions associated with the inversion-related $\pi$-stacked chloroquine molecules (Section 3.2.1.2). For the sake of comparison, energies for a couple of neutral CQ molecules placed at the same relative position as their charged $\mathrm{CQH}_{2}{ }^{2+}$ counterparts are given. As expected, in the real crystal the total interaction energies are determined by repulsive electrostatic terms (Table 4). When $E_{\mathrm{es}}$ are expressed as multipolar contributions (Section S3.3, Supporting Information), they are dominated by a large positive $l=0$ term, weakly alleviated by an attractive $-10 \mathrm{kcal} \cdot \mathrm{mol}^{-1}$ correction due to the interaction of the promolecular part of the density (Table S10, Supporting Information). If the $l=0$ electrostatic term is further decomposed into summations of atomic Mulliken charges (Table 5) of mutually interacting moieties, the relative weights of the various functional groups in determining the gross part of the electrostatic energy can be appreciated. It turns out that the facing quinoline rings account for $+38.5 \%$ of the total destabilization energy (Table 5). The contribution of the two long hydrocarbon chains is similar $(+33.1 \%)$, while interactions of quinoline and lateral chains with the neutral secondary amine N9H9 lower the energy by $-40.3 \%$. The largest repulsion $(+68.7 \%)$ arises from cross-interactions between the two charged quinoline rings and the two lateral hydrocarbon chains, as each pair of quinoline-chain functions provides a $+34.4 \%$ destabilizing contribution. Dispersive $\left(E_{\mathrm{d}}\right)$ and exchange-repulsion $\left(E_{\mathrm{r}}\right)$ contributions (Table 4) exactly cancel each other, leaving the repulsion-dominated electrostatic term as the unique actor on the stage of the interaction energy. In this context, the question of whether some kind of $\pi \cdots \pi$ stabilizing interactions are present or not in the title compound spontaneously arises.

$\pi \cdots \pi$ interactions are somewhat elusive, as they derive from a mixture of electrostatic ${ }^{57}$ and van der Waals ${ }^{58}$ terms, which are both strongly local. The first ones entail electron clouds at a very large distance through the $d^{-1}$ functional, while the second ones involve time-dependent perturbations of the whole interacting ED's, which cannot be properly accounted for by ground-state adiabatic DFT methods. ${ }^{59}$ As an approximate estimation of the weight of $\pi \cdots \pi$ interactions in this system, we

Table 5. Charge-Charge $(l=0)$ Coulomb Contributions to the Monopolar $l=0$ Electrostatic Term among Different Functional Groups $^{a}$ in Charged $\left(\mathrm{CQH}_{2}{ }^{2+} \cdots \mathrm{CQH}_{2}{ }^{2+}\right)$ and Neutral (CQ $\left.\cdots \mathrm{CQ}\right)$ Chloroquine Pairs ${ }^{b}$

$\begin{array}{lccccc}\text { system } & \text { quinoline-quinoline } & \text { quinoline-chain } & \text { chain-chain } & \mathrm{N} 9 \mathrm{H} 9 \text { (all) } & \text { total } l=0 \\ \mathrm{CQH}_{2}{ }^{2+} \ldots \mathrm{CQH}_{2}{ }^{2+} & +55.6 & +99.3 & +47.9 & -58.3 & +144.5 \\ \mathrm{CQ} \cdots \mathrm{CQ} & -3.1 & -1.7 & +2.9 & -1.4 & -3.2\end{array}$

${ }^{a}$ Values are given in $\mathrm{kcal} \cdot \mathrm{mol}^{-1}$. ${ }^{\text {“ }}$ Quinoline" stands for the bicyclic condensed aromatic system, with the exclusion of the secondary amine N9H9. "N9H9" means the secondary amine, which remains formally neutral in both the pairs. All the interactions of this group with both the molecular backbones are summed up. "Chain" collects all the atoms belonging to the hydrocarbon chain bonded to N9. See also Figure 1 and Scheme 1. 
analyzed the ECDA energy decomposition for the couple of neutral CQ molecules (last row in Tables 4 and S10, Supporting Information). Although a change in the protonation state of the drug should affect the strength of $\pi \cdots \pi$ interactions, as it influences the electron-withdrawing ability of the ring substituents, ${ }^{60,61}$ once having eliminated the overwhelming electrostatic term due to the net charges we should at least have a qualitative indication of the importance of $\pi \cdots \pi$ stacking in determining the interaction energy. First, we note that repulsion, mostly due to the closeness of the quinoline cores with the lateral chains, completely counteracts the $E_{\mathrm{d}}$ term also in neutral chloroquine. Second, a weakly attractive monopole $l$ $=0$ contribution is brought in by direct interactions between facing quinoline rings (Table 5 and Table S10, Supporting Information), meaning that the mutual arrangement of charges in the facing aromatic systems is favorable, according also to their head-to-tail orientation (Figure $5 b$ ). As shown before, CQ molecules are shifted so that atoms $\mathrm{C} 7$ and $\mathrm{C} 8 \mathrm{~A}$ roughly place themselves in correspondence to the local ED minima associated with the ring critical points of the six-membered ring moieties, further determining a favorable association of charge accumulation and depletion zones. In fact, the $\nabla^{2} \rho$ critical points associated with charge concentration regions (Figure S10, Supporting Information) roughly face the charge depletion zones associated with the $\operatorname{ED}(3,+1)$ rcp's at the center of the reciprocally underlying six-membered ring (Figure $5 \mathrm{~b}$ and S10, Supporting Information). Such Laplacian features comply with the slightly favorable electrostatic arrangement predicted by the analysis of the distributions of atomic charges.

Finally, following Zhikol et al., ${ }^{54}$ we also tried to estimate the stabilization energy from the topological parameters of the cage critical points (ccp's) associated with the stacking motif (Table 6). To this end, we applied their empirical functional to have a

Table 6. Topological Parameters (Atomic Units) of Relevant Symmetry-Independent ED Cage Critical Points (ccp's) and

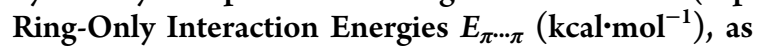
Estimated from Equation 1, for the Stacked Charged $\left(\mathrm{CQH}_{2}{ }^{2+} \ldots \mathrm{CQH}_{2}{ }^{2+}\right)$ and Neutral (CQ..CC) Chloroquine Pairs in Solid-State Geometry at $T=103 \mathrm{~K}$ (Figure 5)

\begin{tabular}{lcccc}
\multicolumn{1}{c}{ system } & $\mathrm{C}$ & $\rho_{\text {ccp }}$ & $\nabla^{2} \rho_{\text {ccp }}$ & $E_{\pi \cdots \pi}$ \\
$\mathrm{CQH}_{2}{ }^{2+} \ldots \mathrm{CQH}_{2}{ }^{2+}$ & -0.94555 & 0.00169 & 0.01257 & $+4.4(6)$ \\
$\mathrm{CQ} \cdots \mathrm{CQ}$ & -0.93716 & 0.00169 & 0.01255 & $+4.3(6)$ \\
\hline
\end{tabular}

rough estimate of the contribution of facing aromatic rings to the interaction energy, $E_{\pi \cdots \pi}$ (all quantities in atomic units):

$$
\begin{aligned}
E_{\pi \cdots \pi}= & \sum_{i} C_{i}\left\{1.214(14) \cdot \nabla^{2} \rho_{i}-131.6(3.9) \cdot\left(\nabla^{2} \rho_{i}\right)^{2}\right. \\
& \left.+635(84) \rho_{i}^{2}\right\}
\end{aligned}
$$

In (1), $\nabla^{2} \rho_{\mathrm{i}}$ is the Laplacian of the charge density $\rho_{\mathrm{i}}$ at the $i$ th ccp and $C_{\mathrm{i}}$ is the cosine of the angle between the ccp and the two rcp's of its closest aromatic rings. The summation includes all the ccp's related to the ring stacking, which are located roughly halfway between the sandwiched quinoline rings.

The functional (1) was developed through a least-squares fitting procedure to empirically reproduce the MP2 interaction energies of simple benzene dimers in various stacking geometries. Chloroquine has a far more complex topology: for example, two symmetry-related ccp's are present in the stacking region of the drug. Each of them contributes for
$+2.2(4) \mathrm{kcal} \cdot \mathrm{mol}^{-1}$ to $E_{\pi \cdots \pi}$, a value close to the Zhikol et al. ${ }^{54}$ estimate $(+2.4)$ for close benzene rings in a similarly staggered conformation. When neutral chloroquine molecules are considered, both the charge density and its Laplacian at the ccp are much alike those for the charged pair, resulting in a similar unfavorable $E_{\pi \cdots \pi}$ estimate.

In conclusion, some attractive contributions, essentially electrostatic in nature, are present in the neutral CQ $\cdots$ CQ $\pi$ stacked pair. The latter arise from a leading promolecular term ( - $10 \mathrm{kcal} \cdot \mathrm{mol}^{-1}$, Table S10, Supporting Information), which depends on the interaction geometry and enables a significant superposition of the undistorted promolecular densities, as well as from a favorable arrangement of atomic charges of the facing quinoline rings. However, granted that Coulomb-driven attractive interactions are established in this pair, mirroring some kind of stabilizing $\pi \cdots \pi$ motif, they are also very weak; for sure, they are completely overwhelmed in the charged $\mathrm{CQH}_{2}{ }^{2+} \cdots \mathrm{CQH}_{2}{ }^{2+}$ pair. We also expect that any $\pi \cdots \pi$ motif in solution should be even less significant as a possible structure-driving interaction. Indeed, a static periodic arrangement with molecules kept in close contact by the crystal packing, despite their formal charges, should provide the most possibly favorable interaction mode.

3.3. A Model for Chloroquine/Heme Interaction. In the acidic $(\mathrm{pH} \sim 5.0)$ digestive vacuole (DV) of Plasmodium, the majority of free heme released from the digestion of hemoglobin is present as monoprotonated neutral hematin, (FePPIX $\left.\left(\mathrm{H}_{2} \mathrm{O}\right) \mathrm{H}\right)$, with a water molecule weakly coordinated to $\mathrm{Fe}$ and to one of the two side propionic acid functions of protoporphyrin IX $\left(\mathrm{p} K_{\mathrm{a}}=4.3\right.$ and 5.5), predominantly undissociated. ${ }^{12,62}$ We recently demonstrated ${ }^{12}$ that a ligand exchange acid-base reaction is thermodynamically possible in the DV,

$$
\begin{aligned}
& \operatorname{FePPIX}\left(\mathrm{H}_{2} \mathrm{O}\right) \mathrm{H}+\mathrm{CQH}_{2}^{2+} \\
& \rightarrow \operatorname{FePPIX}(\mathrm{CQH}) \mathrm{H}^{+}+\mathrm{H}_{3} \mathrm{O}^{+}
\end{aligned}
$$

and one which creates a direct $\mathrm{Fe}-\mathrm{N}$ bond with the quinoline nitrogen $\mathrm{N} 1$. The resulting $\mathrm{FePPIX}(\mathrm{CQH}) \mathrm{H}^{+}$complex is stabilized by strong CAHBs between the charged propionate group of the protoporphyrin and the tertiary protonated amine, $\mathrm{N14}$, in the CQ hydrocarbon chain. This structure is compatible with EXAFS findings in aqueous acidic solutions at room temperature. ${ }^{12}$ To explore analogies and differences with respect to crystalline chloroquine on the same ground, the iron complex was reoptimized in vacuo at the m-GGA B3LYP/ pob-TZVP theory level. The curvatures of the potential energy hypersurface at the stationary point were evaluated to ensure that a true minimum geometry (Figure 8) was effectively found. The minimum geometry is similar to that formerly predicted at the lower GGA B3LYP 6-311G(p,d) level of theory. ${ }^{12}$ The most striking difference resides in the orientation of the quinoline ring, which here is folded toward the protoporphyrin aromatic system, so that the least-squares planes associated with these moieties make a dihedral angle as low as $17.3^{\circ}$. For the sake of comparison, the same angle amounts to $78.7^{\circ}$ in the GGA-optimized structure, where the two aromatic systems are almost orthogonal to each other. This difference is likely due to the introduction of the empirical dispersion term in the $m$-GGA functional, which favors conformations that maximize the contact surface between van der Waals envelopes of the interacting species. 


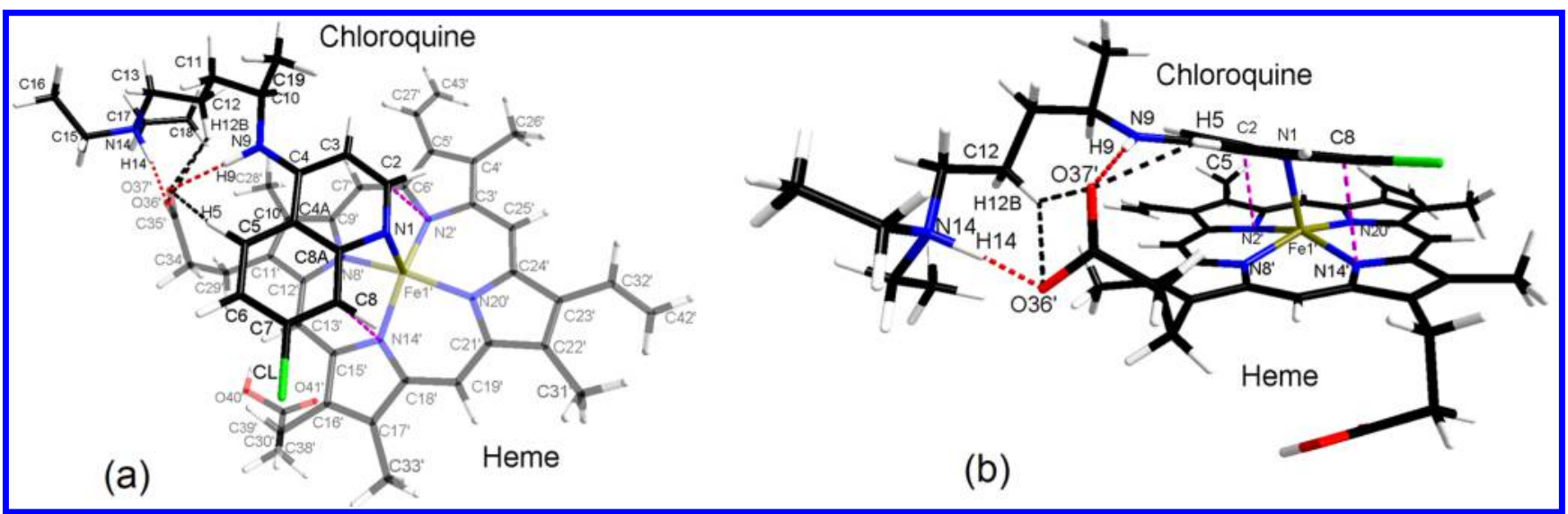

Figure 8. (a) Geometry of a possible heme/chloroquine adduct, as predicted by a $m$-GGA B3LYP/pob-TZVP quantum calculation in vacuo, with the atom numbering scheme (primed labels refer to heme). Dashed lines represent $\mathrm{NH} \cdots \mathrm{O}$ CAHBs (red), $\mathrm{CH} \cdots \mathrm{O}$ (black), and C $\cdots \mathrm{N}$ (purple) contacts associated with a topological AIL. Shaded features refer to atoms below the plane of the picture. (b) Same as (a), with the quinoline plane perpendicular to the plane of the picture to highlight the relevant intermolecular contacts. Cartesian coordinates of the adduct can be found in Table S11, Supporting Information.

Table 7. Geometrical, Topological and HB Energy Properties of Intermolecular Atom-Atom Contacts Associated to AILs in the Chloroquine-Heme Adduct (Figure 8) ${ }^{a}$

\begin{tabular}{|c|c|c|c|c|c|c|c|}
\hline$X \cdots Y$ & $d_{\mathrm{X} \cdots \mathrm{Y}} / \AA$ & $\rho_{\mathrm{bcp}} / \mathrm{au}$ & $\nabla^{2} \rho_{\mathrm{bcp}} / \mathrm{au}$ & 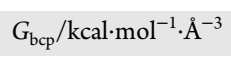 & $V_{\mathrm{bcp}} / \mathrm{kcal} \cdot \mathrm{mol}^{-1} \cdot \AA^{-3}$ & $\mathrm{BD} / \mathrm{au}$ & $E(2)^{b} / \mathrm{kcal} \cdot \mathrm{mol}^{-1}$ \\
\hline N9H9 …O37' & 1.869 & 0.029 & 0.107 & 15.8 & -14.9 & +0.05 & 4.0 \\
\hline $\mathrm{N} 14 \mathrm{H} 14 \cdots \mathrm{O} 36^{\prime}$ & 1.429 & 0.096 & 0.129 & 45.0 & -69.8 & -0.41 & 62.8 \\
\hline 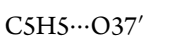 & 2.299 & 0.015 & 0.051 & 6.8 & -5.5 & +0.14 & 1.7 \\
\hline $\mathrm{C} 12 \mathrm{H} 12 \mathrm{~B} \cdots \mathrm{O} 36^{\prime}$ & 2.522 & 0.011 & 0.039 & 5.2 & -4.4 & +0.13 & 0.2 \\
\hline $\mathrm{C} 12 \mathrm{H} 12 \mathrm{~B} \cdots \mathrm{O} 37^{\prime}$ & 2.433 & 0.014 & 0.049 & 6.7 & -5.8 & +0.11 & 0.2 \\
\hline $\mathrm{C} 2 \cdots \mathrm{N} 2^{\prime}$ & 2.902 & 0.015 & 0.055 & 7.5 & -6.5 & +0.11 & \\
\hline $\mathrm{C} 8 \cdots \mathrm{N} 14^{\prime}$ & 2.958 & 0.013 & 0.046 & 6.4 & -5.6 & +0.10 & \\
\hline $\mathrm{N} 1 \cdots \mathrm{Fe} 1$ & 2.100 & 0.075 & 0.232 & 48.2 & -60.0 & -0.25 & // \\
\hline
\end{tabular}

In any case, some similarities emerge between the CQ coordination network in the studied crystal and in the heme/ CQ adduct. First, the BSSE- and relaxation-corrected interaction energy between the Fe-protoporphyrin-IX and single-protonated $\mathrm{CQH}^{+}$species in the complex amounts to $\sim-106 \mathrm{kcal} \cdot \mathrm{mol}^{-1}$, a value comparable with that found for the $\mathrm{CQH}_{2}{ }^{2+} \ldots \mathrm{H}_{2} \mathrm{PO}_{4}{ }^{-}$pair (III) (Table 4). Second, the highly stabilizing $\mathrm{N} 9-\mathrm{H} 9 \cdots \mathrm{O}^{-}$and $\mathrm{N} 14-\mathrm{H} 14 \cdots \mathrm{O}^{-}$contacts are conserved both in the chloroquine crystal and in the drugheme complex (Tables 2, 4, and 7). Moreover, the topological parameters at the bcp of the AIL involving the N9H9 HB donor are nearly identical in the two systems. However, the NBO E(2) estimates reveal that this $\mathrm{HB}$ is expected to be at least 3 times more stable in the crystal. This discrepancy reflects the fact that point topological descriptors provide an inherently local picture of the ED properties, which do neither necessarily nor easily correlate with the interaction energies. ${ }^{45,63}$ Mulliken charges of $\mathrm{N} 9-\mathrm{H} 9 \cdots \mathrm{O}$ atoms within the $\mathrm{CQH}_{2}{ }^{2+} \ldots \mathrm{CQH}_{2}{ }^{2+}$ pair extracted from the crystal and the chloroquine/heme adduct (see Table S12, Supporting Information) show that N9 and $\mathrm{H} 9$ atoms remain similar, while the negative $\mathrm{O}$ charge decreases in magnitude from $|0.91|$ in the phosphate to $|0.67|$ in the heme propionate, leading to a less favorable Coulomb contribution. In summary, the energy of the N9-H9 $\cdots \mathrm{O}^{-} \mathrm{HB}$ significantly changes without affecting the local properties of the charge density. On the contrary, the $\mathrm{N} 14-\mathrm{H} 14 \cdots \mathrm{O}^{-} \mathrm{CAHB}$ is strongly reinforced in the drug-heme adduct, as it can be appreciated by the much shorter $(\sim 1.4 \AA) \mathrm{H} \cdots \mathrm{O}$ distance and by the large and negative $\mathrm{BD}$ parameter (Table 7), which implies a stronger tendency toward electron sharing. This contact alone provides the $45.7 \%$ of the stabilization energy, while weaker $\mathrm{CH} \cdots \mathrm{O}^{-}$interactions yield poorly significant stabilizing contributions (Table 7). Also the electrostatic weight becomes more favorable in this interaction, as the lower absolute charge of the oxygen propionate in the heme/CQ complex is contrasted by the increased $\mathrm{H} 14$ positive charge and the decreased $\mathrm{H} \cdots \mathrm{O}$ distance (Table 7 and Tables S6, S12, Supporting Information).

The close proximity of the quinoline ring to the protoporphyrin system implies the appearance of some weak AILs connecting facing $\mathrm{C}$ atoms of the drug with $\mathrm{N}$ atoms of the heme (Table 7).

The N1-Fe1 AIL (last row of Table 7) is associated with the coordinative bond with the basic N1 center of the quinoline ring. It thus shows markedly different topological parameters with respect to the weaker $\mathrm{C} 2 \cdots \mathrm{N} 2{ }^{\prime}$ and $\mathrm{C} 8 \cdots \mathrm{N} 14^{\prime}$ contacts. Because of the different approaching mode of the two molecules in the heme adduct with respect to the solid state, the stacking arrangement is also topologically different: for example, in the adduct, $\mathrm{C} 8 \mathrm{~A}$ is too far from $\mathrm{N}^{\prime}$ to determine a significant direct through-bond interaction (Table 7, Figure 8). However, $\mathrm{C} 2 \cdots \mathrm{N} 22^{\prime}$ and $\mathrm{C} 8 \cdots \mathrm{N} 14^{\prime}$ AILs invariably imply $\rho_{\text {bcp }}$ estimates at least 1 order of magnitude higher than for the analogue $\mathrm{C} \cdots \mathrm{N}$ contacts in the crystal (Table 3 ). Accordingly, the energy densities $G_{\mathrm{b}}$ and $V_{\mathrm{b}}$ are from 2 to 3 times larger in magnitude, indicating that these interactions are stronger in the 
complex. Likewise in the title compound two cage critical points are present near the protoporphyrine IX aromatic system (Table S13, Supporting Information), exploiting a total $E_{\pi \cdots \pi}$ stabilization as large as $-31(2) \mathrm{kcal} \cdot \mathrm{mol}^{-1}$, according to the functional by Zhikol et al. ${ }^{54}$ (Section 3.2.2.2). The latter energy corresponds to $\sim 22.7 \%$ of the total interaction energy, while $E_{\pi \cdots \pi}$ was significantly closer to zero in the solid-state stacked chloroquine (Table 6). This result was somehow expected on the basis of the shorter average distance of the quinoline ring from the least-squares plane of the protoporphyrin (3.1(1) $\AA$, vs $\sim 3.4 \AA$ for the $\mathrm{CQH}_{2}{ }^{2+} \ldots \mathrm{CQH}_{2}{ }^{2+}$ system, see Section 3.2.1).

Note that the two interacting rings are not parallel in the drug-heme adduct. This arrangement is not uncommon per se, as various structures are known which are tilted by some degrees with respect to the stacking axis, such as for example $\beta$ cyclodextrins. ${ }^{64}$ However, the functional given by eq 1 was developed from a training set of planar, parallel benzene rings. Therefore, it might be not as accurate in such a complex system, leading to a likely overestimated $E_{\pi \cdots \pi}$ energy. Indeed, part of the delocalized $\pi$ system of the quinoline moiety is kept away from the underlying protoporphyrin cycle. For sure, both geometrical and topological parameters agree in pointing out that stacking interactions between the two cycles are stronger in the adduct than in the $\mathrm{CQH}_{2}{ }^{2+} \ldots \mathrm{CQH}_{2}{ }^{2+}$ solid state pair, even though they are considerably weaker than the CAHBs formed by the tertiary amine group of chloroquine.

Figure 9 shows the RDG plot for the FePPIX $(\mathrm{CQH}) \mathrm{H}^{+}$ adduct. Five sharp lenticular isosurfaces exploiting markedly

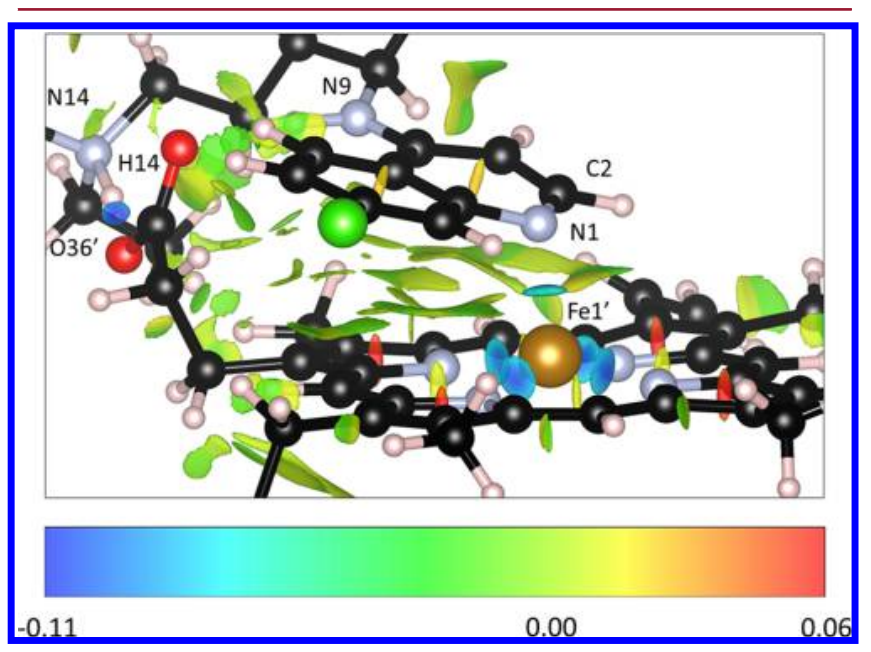

Figure 9. $\operatorname{Sign}\left(\lambda_{2}\right) \cdot \rho$ plotted onto RDG isosurfaces (value $=0.34$ ) in the internuclear region between the facing aromatic rings in the FePPIX $(\mathrm{CQH}) \mathrm{H}^{+}$adduct. Values on the chromatic scale are given in atomic units.

negative $\operatorname{sign}\left(\lambda_{2}\right) \cdot \rho$ values are appreciable around $\mathrm{Fe}$, corresponding to the five $\mathrm{Fe}-\mathrm{N}$ coordinative bonds set up by the metal ion with the protoporphyrin and quinoline systems. Interestingly, a similar feature appears between $\mathrm{H} 14$ and O36', indicating that, as anticipated by the point topological descriptors (Table 7), the N14-H14‥O36' CAHB is extremely strong and bears features comparable to the coordinative $\mathrm{N}-\mathrm{Fe}$ interactions.

On the other hand, the region sandwiched by the interacting aromatic systems is dominated by unstructured large and flat RDG isosurfaces, bearing negative $\operatorname{sign}\left(\lambda_{2}\right) \cdot \rho$ values. When a pure dispersive-repulsive balance is established in the intermolecular region, RDG surfaces similar in shape appear, but they are invariably associated with a rather indeterminate sign of $\lambda_{2}$ and to $\rho$ values much closer to zero. ${ }^{41}$ In the present case some kind of attractive $\pi \cdots \pi$ interactions seem to occur and are significantly stronger than those established between facing charged chloroquine molecules, where the $\operatorname{sign}\left(\lambda_{2}\right) \cdot \rho$ function plotted onto the RDG isosurfaces is invariably positive or very close to zero (Figure 6a).

\section{CONCLUSIONS}

In this work, a combined experimental and theoretical study was carried out on the chloroquine dihydrogen phosphate dihydrate salt at $T=103(2) \mathrm{K}$. This investigation allowed us to explore the NCI network, which determines how the drug interacts with its neighborhood in the solid state, as a model for the drug-substrate recognition process. Likely, the same structure-determining factors are active also in solution, where they are controlled by the Boltzmann averages over thermally accessible rotational and vibrational states rather than by the crystal packing.

Any debate on the structure of the heme/CQ adduct should explicitly take into account the chemical environment where it forms, and in particular the $\mathrm{pH}$. In the acidic vacuolar environment ( $\mathrm{pH}=5.0$ or less), the acid-base equilibria are shifted, on the one hand, toward fully protonated chloroquine, and, on the other hand, toward neutral hematin, where one water molecule occupies the axial position of the pentacoordinated iron ion and one propionate group is dissociated. Although in this state hematin is overall neutral, the center of the protoporphyrin IX ring remains positively charged, as oxidized $\mathrm{Fe}$ (III) prevails over the two negative charges borne by the equatorial pyrrole functions. Thus, interactions between the quinoline system of $\mathrm{CQH}_{2}^{2+}$ and the FePPIX scaffold should be repulsive, rather than attractive, in agreement to what observed for close-packed, $\pi \cdots \pi$ stacked, and charged chloroquine molecules in the crystal. Even though some weak electrostatic attractive interactions might be present, due to local anisotropies in the ED distribution, they are totally overwhelmed by Coulomb repulsions between the protonated quinoline Nitrogen and the unscreened $\mathrm{Fe}^{3+}$ ion. Such a repulsive arrangement is even more unlike in solution, where molecules are translationally free and can alleviate electrostatic repulsions by simply keep moving.

The picture is quite different when a coordinative $\mathrm{Fe}-\mathrm{N}$ bond is allowed to form. Now no net electrostatic repulsion exists, and the direct bond between chloroquine and heme keeps the quinoline system very close to the protoporphyrin ring. From topological, structural, and energetic viewpoints, $\pi \cdots \pi$ interactions become stronger in the heme-drug adduct than in the $\mathrm{CQH}_{2}{ }^{2+} \ldots \mathrm{CQH}_{2}{ }^{2+}$ solid-state pair. Within the limits of our modeling approach, we estimated that they should contribute at most by $\sim 23 \%$ to the total interaction energy. Nevertheless, attractive drug-substrate NCIs, which involve the protonated tertiary amine of chloroquine, seem even more important. As expected, the $\mathrm{CQH}_{2}{ }^{2+} \cdots \mathrm{H}_{2} \mathrm{PO}_{4}{ }^{-}$interactions are responsible for the stable dihydrogen phosphate salt structure. Both the protonated basic functions in the crystal are involved in strong CAHBs with the negatively charged counterions, even though there is not a direct correspondence between the strength of the HBs and the interaction energies of the corresponding molecular pairs. In the heme/chloroquine complex, a single CAHB relating the protonated amine of the drug with the charged propionate function of the proto- 
porphyrin ring accounts for more than $45 \%$ of the interaction energy, confirming its leading importance for stabilizing the adduct.

In conclusion, strong CAHBs and, to a minor extent, $\pi \cdots \pi$ interactions cooperate in determining the drug-substrate recognition mode in an acidic chemical environment. The stable heme/chloroquine interaction geometry here proposed is still compatible with our previous EXAFS findings. ${ }^{12}$ Most important, it might reconcile the contemporary presence of a stable $\pi \cdots \pi$ motif, hypothesized by several spectroscopic techniques, with a direct $\mathrm{Fe}-\mathrm{N}$ coordinative bond, as suggested by EXAFS and NMR evidence. It follows that the subsequent chemical features are all required to explain the heme-binding ability of chloroquine: (i) a hydrocarbon chain long enough to reach the propionic functions of the protoporphyrin system; (ii) a protonable strong basic function at the end of the chain; (iii) a less strong Lewis base at the other end of the molecule, able to saturate the axial position of the metal center, and (iv) a flat aromatic system to further stabilize the complex through $\pi \cdots \pi$ interactions.

Further studies are in order to investigate what chemical features allow the drug to be recognized by the protein transporters at the core of the forced efflux resistance mechanism. Hopefully, some features of the pharmacophore might be engineered to develop novel cheap CQ-based antiplasmodials without hampering the heme-binding ability of the parent compound.

\section{ASSOCIATED CONTENT}

\section{S Supporting Information}

The Supporting Information is available free of charge on the ACS Publications website at DOI: 10.1021/acs.cgd.6b01069.

Section S1: Technical details on the solid-state DFT optimization procedure. Section S2: Crystallographic analysis of the title compound. On the nature of the disorder in the title compound. Hamilton test. Solid-state molecular dynamics. On the validity of the proposed DFT model. Section 3: Intermolecular interactions in the crystal. Topology. Comments on the ECDA decomposition vs DFT energy estimates. Multipolar decomposition of $E_{\mathrm{es}}$. Details of the $\mathrm{CQH}_{2}{ }^{2+} \cdots \mathrm{H}_{2} \mathrm{PO}_{4}{ }^{-}$ interaction energetics. Section S4: DFT geometries, charges and topological parameters (PDF)

\section{Accession Codes}

CCDC 1471834 and 1494003 contain the supplementary crystallographic data for this paper. These data can be obtained free of charge via www.ccdc.cam.ac.uk/data request/cif, or by emailing data_request@ccdc.cam.ac.uk, or by contacting The Cambridge Crystallographic Data Centre, 12 Union Road, Cambridge CB2 1EZ, UK; fax: +44 1223336033.

\section{AUTHOR INFORMATION}

\section{Corresponding Author}

*E-mail: leonardo.lopresti@unimi.it. Phone: +39-02-50314252. Fax: +39-02-50314300.

\section{Notes}

The authors declare no competing financial interest.

\section{ACKNOWLEDGMENTS}

Three of us (G.M., C.G., and L.L.P.) thank the Danish National Research Foundation for funding Ph.D. positions in our group through the Center for Materials Crystallography (DNRF93).
Partial funding provided by Università degli Studi di Milano (Unimi) through the Development Plan of Athenaeum grantline B1 (UNIAGI 17777) was appreciated. We also thank the CINECA Italian supercomputing center for providing computational time through the ISCRA $\mathrm{C}$ initiative (Project MODELPIP) and convention with Unimi. Finally, technical assistance by Mr. P. Colombo is warmly acknowledged.

\section{REFERENCES}

(1) Warhurst, D. C.; Steele, J. C. P.; Adagu, I. S.; Craig, J. C.; Cullander, C. I. Antimicrob. Chemother. 2003, 52, 188-193.

(2) Slater, A. F. G.; Swiggard, W. J.; Orton, B. R.; Flitter, W. D.; Goldberg, D. E.; Cerami, A.; Henderson, G. B. Proc. Natl. Acad. Sci. U. S. A. 1991, 88, 325-329.

(3) Leed, A.; DuBay, K.; Ursos, L. M. B.; Sears, D.; de Dios, A. C.; Roepe, P. D. Biochemistry 2002, 41, 10245-10255.

(4) de Dios, A. C.; Tycko, R.; Ursos, L. M. B.; Roepe, P. D. I. Phys. Chem. A 2003, 107, 5821-5825.

(5) Gildenhuys, J.; le Roex, T.; Egan, T. J.; De Villiers, K. A. L. Am. Chem. Soc. 2013, 135, 1037-1047.

(6) Kořený, L.; Oborník, M.; Lukeš, J. PLoS Pathog. 2013, 9 (e1003088), 1-6.

(7) Kumar, S.; Bandyopadhyay, U. Toxicol. Lett. 2005, 157, 175-188.

(8) Dascombe, M. J.; Drew, M. G. B.; Morris, H.; Wilairat, P.; Auparakkitanon, S.; Moule, W. A.; Alizadeh-Shekalgourabi, S.; Evans, P. G.; Lloyd, M.; Dyas, A. M.; Carr, P.; Ismail, F. M. D. L. Med. Chem. 2005, 48, 5423-5436.

(9) Walczak, M. S.; Lawniczak-Jablonska, K.; Wolska, A.; Sienkiewicz, A.; Suarez, L.; Kosar, A. J.; Bohle, D. S. L. Phvs. Chem. B 2011, 115, $1145-1150$.

(10) Portela, C.; Afonso, C. M. M.; Pinto, M. M. M.; Ramos, M. I. FEBS Lett. 2003, 547, 217-222.

(11) Asghari-Khiavi, M.; Vongsvivut, J.; Perepichka, I.; Mechler, A.; Wood, B. R.; McNaughton, D.; Bohle, D. S. I. Inorg. Biochem. 2011, $105,1662-1169$.

(12) Macetti, G.; Rizzato, S.; Beghi, F.; Silvestrini, L.; Lo Presti, L. Phvs. Scr. 2016, 91 (2), 023001.

(13) Egan, T. J. I. Inorg. Biochem. 2006, 100, 916-926.

(14) Guantai, E.; Chibale, K. Curr. Drug Delivery 2010, 7, 312-323.

(15) Chinappi, M.; Via, A.; Marcatili, P.; Tramontano, A. PLoS One 2010, 5 (11), e14064.

(16) Robert, M.-C.; Vidal, O.; Garda-Ruiz, J.-M.; Otalora, F. In Crystallization of Nucleic Acids and Proteins. A Practical Approach; Ducruix, A., Giegé, R., Eds.; IRL Press: Oxford1992; pp 149-176.

(17) SAINT+; Bruker AXS Inc.: Madison, Wisconsin, USA, 2012.

(18) SADABS; Bruker AXS Inc., Madison, Wisconsin, USA, 2014

(19) XPREP; Bruker AXS Inc., Madison, Wisconsin, USA, 2014.

(20) Sheldrick, G. M. Acta Crystallogr., Sect. A: Found. Adv. 2015, 71, $3-8$

(21) Dovesi, R.; Saunders, V. R.; Roetti, C.; Orlando, R.; ZicovichWilson, C. M.; Pascale, F.; Civalleri, B.; Doll, K.; Harrison, N. M.; Bush, I. J.; D’Arco, P.; Llunell, M.; Causà, M.; Noël, Y. CRYSTAL14 User's Manual; University of Torino, Torino, Italy, 2014.

(22) Gatti, C.; Casassa, S. TOPOND14 User's Manual; CNR-ISTM of Milano, Milano, 2014.

(23) Bader, R. F. W. Atoms in Molecules: A Quantum Theory, International Series of Monographs on Chemistry 22; Oxford Science Publications: Oxford, UK, 1990.

(24) Matta, C. F.; Boyd, R. J. The Quantum Theory of Atoms in Molecules: From Solid State to DNA and Drug Desion; Wiley-VCH Verlag GmbH \& Co. KGaA: Weinheim, Germany, 2007.

(25) Gatti, C.; Macchi, P. Modern Charge Densitv Analvsis; Springer: Dordrecht, 2012.

(26) Peintinger, M. F.; Oliveira, D. V.; Bredow, T. L. Comput. Chem. 2013, 34, 451-459.

(27) Frisch, M. J.; Trucks, G. W.; Schlegel, H. B.; Scuseria, G. E.; Robb, M. A.; Cheeseman, J. R.; Scalmani, G.; Barone, V. et al. Gaussian 09, Revision E.01; Gaussian, Inc.: Wallingford CT, USA, 2009. 
(28) Boys, S. F.; Bernardi, F. Mol. Phvs. 1970, 19, 553-566.

(29) Lo Presti, L.; Ellern, A.; Destro, R.; Lunelli, B. I.Phys. Chem. A 2009, 113, 3186-3196.

(30) Lo Presti, L.; Soave, R.; Longhi, M.; Ortoleva, E. Acta Crystallogr., Sect. B: Struct. Sci. 2010, 66, 527-543.

(31) Lo Presti, L.; Sist, M.; Loconte, L.; Pinto, A.; Tamborini, L.; Gatti, C. Cryst. Growth Des. 2014, 14, 5822-5833.

(32) Gavezzotti, A.; Lo Presti, L. Cryst. Growth Des. 2015, 15, 37923803.

(33) Spackman, M. A.; Weber, H. P.; Craven, B. M. I.Am. Chem. Soc. 1988, 110, 775-782.

(34) Spackman, M. A. I. Chem. Phvs. 1986, 85, 6579-6586.

(35) Spackman, M. A. I. Chem. Phvs. 1986, 85, 6587-6601.

(36) Spackman, M. A. I. Phvs. Chem. 1987, 91, 3179-3186.

(37) Spackman, M. A. Keynote Lecture KN23.28 at IUCR2005, Book of Abstracts, Firenze, Italy; 2005; p C5.

(38) Barzaghi, M PAMoC [Version 2010-07-27], Online User's Manual; CNR-ISTM, Institute of Molecular Science and Technologies: Milano, Italy, 2010; http://www.istm.cnr.it/pamoc/.

(39) Glendening, E. D; Reed, A. E.; Carpenter, J. E.; Weinhold, F. NBO Version 3.1.

(40) Johnson, E. R.; Keinan, S.; Mori-Sanchez, P.; Contreras-García, J.; Cohen, A. J.; Yang, W. L. Am. Chem. Soc. 2010, 132, 6498-6506.

(41) Saleh, G.; Gatti, C.; Lo Presti, L.; Contreras-García, J. Chem. Eur. I. 2012, 18, 15523-15536.

(42) Saleh, G.; Gatti, C.; Lo Presti, L.; Ceresoli, D. L. Appl. Crystallogr. 2013, 46, 1513-1517.

(43) Preston, H. S.; Stewart, J. M. I. Chem. Soc. D 1970, 1142-1143.

(44) Karle, J. M.; Karle, I. L. Acta Crystallogr., Sect. C: Cryst. Struct. Commun. 1988, 44, 1605-1608.

(45) Gavezzotti, A.; Lo Presti, L. Cryst. Growth Des. 2016, 16, 29522962.

(46) Destro, R.; Soave, R.; Barzaghi, M.; Lo Presti, L. Chem. - Eur. I. 2005, 11, 4621-4634.

(47) Saleh, G.; Soave, R.; Lo Presti, L.; Destro, R. Chem. - Eur. I. 2013, 19, 3490-3503.

(48) Politzer, P.; Murray, J. S.; Peralta-Inga, Z. Int. I. Quantum Chem. 2001, 85, 676-684.

(49) Bondi, A. I. Phvs. Chem. 1964, 68, 441-451.

(50) Espinosa, E.; Alkorta, I.; Elguero, J.; Molins, E. I. Chem. Phvs. 2002, 117, 5529-5542.

(51) Boto, R. A.; Contreras-García, J.; Tierny, J.; Piquemal, J.-P. Mol. Phvs. 2016, 114, 1406-1414.

(52) Matta, C. F.; Castillo, N.; Boyd, R. J. I. Phvs. Chem. B 2006, 110 (1), 563-578.

(53) Churchill, C. D. M.; Rutledge, L. R.; Wetmore, S. D. Phys. Chem. Chem. Phvs. 2010, 12, 14515-14526.

(54) Zhikol, P. A.; Shishkin, O. V.; Lyssenko, K. A.; Leszczynski, J. L. Chem. Phys. 2005, 122, 144104.

(55) Palusiak, M.; Bankiewicz, B. Comput. Theor. Chem. 2011, 966, $113-119$.

(56) A self-consistent least-squares fit was carried out, taking into account data with $d>10 \AA$. In particular, points showing least-squares residuals higher than 2 times their average standard deviation, $<\sigma\left(E_{\text {int,DFT }}\right)>$, were repeatedly excluded from the fit until convergence was achieved. Note that $10 \AA$ is more or less the maximum extension of the chloroquine molecule when it folds in the crystal. Accordingly, it is also the distance below which pro-pro and pro-def terms are significant.

(57) Hunter, C. A.; Sanders, J. K. M. I. Am. Chem. Soc. 1990, 112, 5525-5534.

(58) Ringer, A. L.; Sinnokrot, M. O.; Lively, R. P.; Sherrill, C. D. Chem. - Eur. I. 2006, 12, 3821-3828.

(59) Sholl, D. S.; Steckel, J. A. Density Functional Theorv: A Practical Introduction; Wiley: New York, 2009; p 29.

(60) Sinnokrot, M. O.; Sherrill, C. D. L. Phys. Chem. A 2006, 110, 10656-10668.

(61) Wheeler, S. E.; Houk, K. N. I. Am. Chem. Soc. 2008, 130, 10854-10855.
(62) Durrant, M. C. Dalton Trans. 2014, 43, 9754-9765.

(63) Spackman, M. A. Cryst. Growth Des. 2015, 15, 5624-5628.

(64) Herbstein, F. H. Crustalline Molecular Complexes and Compounds: Structures and Principles; Oxford University Press: Oxford, UK, 2005; Vol. 1., p 113. 